\title{
The Origin of the Metal-Poor Common Proper Motion Pair HD 134439/134440: Insights from New Elemental Abundances
}

\author{
Yu Chen and Jeremy R. King \\ Department of Physics and Astronomy, 118 Kinard Lab, Clemson University, Clemson, SC \\ 29634-0978 \\ yuc@clemson.edu jking2@clemson.edu \\ and \\ Ann M. Boesgaard ${ }^{\dagger}$ \\ Institute for Astronomy, 2680 Woodlawn Drive, Honolulu, HI 96822 \\ boes@ifa.hawaii.edu
}

\begin{abstract}
The low $[\alpha / \mathrm{Fe}]$ ratio in the metal-poor $([\mathrm{Fe} / \mathrm{H}] \sim-1.50)$ common proper motion pair HD 134439 and HD 134440 has been variously attributed to chemical evolution in an extragalactic environment with an irregular star formation history, planetessimal accretion, and formation in an environment with an unusually high dust-to-gas ratio. We explore these various putative origins using $\mathrm{CNO}, \mathrm{Be}, \mathrm{Ag}$, and $\mathrm{Eu}$ abundances derived from high-resolution near-UV Keck/HIRES spectroscopy. While we confirm a previously suggested correlation between elemental abundance ratios and condensation temperature at the $95 \%$ confidence level, these ratios lie within the continuum of values manifested by extant dSph data. We argue that the most plausible origin of our stars' distinctive abundance distribution relative to the Galactic halo field is formation in an environment chemically dominated by products of Type II SN of low progenitor mass; such a progenitor mass bias has been previously suggested as an explanation of low $\alpha$-element ratios of dSph stars. The proper motion pair's heavy-to-light $n$-capture element ratio, which is $\geq 0.3-0.5$ dex lower than in the Galactic halo field and $\mathrm{dSph}$ stars, is discussed in the context of the truncated $r$-process, phenomenlogical $n$-capture production models, and $\alpha$-rich freezeout in a high neutron excess environment; the latter simultaneously provides an attractive explanation of the difference in [Ca, Ti/O,Mg, $\mathrm{Si}$ ] ratio in HD 134439/134440 compared to in situ dSph stars.
\end{abstract}

Subject headings: Stars

\footnotetext{
${ }^{\dagger}$ Visiting Astronomer, W.M KECK Observatory, which is operated as a scientific partnership among the California Institute of Technology, the University of California and the National Aeronautics and Space Administration. The Observatory was made possible by the generous financial support of the W.M. Keck Foundation.
} 


\section{INTRODUCTION}

The outcome of the Eggen et al. (1962) rapid monolithic collapse (ELS) model is an old metalpoor Galactic halo with enhanced $[\alpha / \mathrm{Fe}]$ values, and a young metal-rich disk with near-solar $[\alpha / \mathrm{Fe}]$. In this picture, stellar $[\alpha / \mathrm{Fe}]$ values diminish as a function of Galactic time as metallicity increases from nucleosynthetic feedback that is a sensitive function of stellar mass. However, stars in the Galaxy do not strictly follow the simple trend expected by ELS model. Carney et al. (1997) reported the low metallicity $([\mathrm{Fe} / \mathrm{H}]=-1.86)$ star $\mathrm{BD}+80^{\circ} 245$ to have an abnormally low average $[\alpha / \mathrm{Fe}] \sim-0.3$. CS 22966-043, a blue metal-poor SX Phe variable star, exhibits unusual $\alpha$-element deficiencies (Preston \& Sneden 2000). Other examples include Nissen \& Schuster's (1997) sample of low- $\alpha$ halo stars with large apogalactocentric distances, the expanded Nissen \& Schuster (2010) sample of low- $\alpha$ halo stars that are weakly bound and evince predominantly retrograde orbits, the young globular clusters Rup 106 and Pal 12 (Brown et al. 1997), and the halo field common proper motion pair HD 134439 and 134440 (King 1997a).

The early ELS picture of Galactic formation is undoubtedly much too simplistic, lacking consideration of a variety of important feedback mechanisms associated with star formation (e.g., Hopkins. Naravanan \& Murrav 2013), the effects of recycling timescale (e.g. De Lucia et al. 2014), the context of cosmological influences of dark matter and energy on Galaxy formation (e.g., Penzo et al. 2014), and the consequences of rapid infall of cool intergalactic gas (e.g., Hopkins, Keres \& Murray 2013). Regardless, the existence of low- $\alpha$ stars suggests that an additional or alternative formation mechanism of the Galactic halo is required to explain these abundance patterns.

By examining color-magnitude diagrams of globular clusters in the Galactic halo, Searle \& Zinn (1978) found a diverse horizontal-branch morphology corresponding to an age spread of $\geq 10^{9}$ years, suggesting the halo formation timescale lasted longer than a free-fall time. Consequently, they suggested the outer halo of the Galaxy may be a product of accreted fragments that formed independently of the Galactic collapse. Indeed, a variety of surveys evince the presence of significant tidal streams (Ibata et al. 2001a) and substructure (Yannv et al. 2000) in the halo that is interpreted as detritus from dwarf galaxy encounters (Ibata et al. 2001b; Johnston et al. 2008). Current hydrodynamic simulations of the formation of late-type spirals naturally produce stellar halos populated by accreted ex-situ stars (e.g. Pillepich, Madau \& Mayer 2014).

This hierarchical picture of Galactic development is supported by additional independent evidence. For example, presolar SiC grains show unexpected Si isotopic ratios (see Zinner 1998) that can be explained by a solar birth environment located in the remains of a merger event with a metal-poor satellite galaxy 5-6 Gyr ago (Clayton 2003). Large pre-solar ${ }^{18} \mathrm{O}$ abundances have also been explained as the result of feedback of SNe outbreaks caused by the merger of a satellite galaxy with the Milky Way (Clayton 2004). Prantzos (2007) shows that the halo metallicity distribution is well reproduced by the merger of a collection of sub-halo satellite systems having various masses.

The properties of present-day dwarf spheroidal (dSph) systems provide evidence of hierarchical formation of the Galactic halo (Menci et al. 2002). dSphs have distinctly different environments 
than the Milky Way-notably their irregular and/or small star formation rates and strong galactic winds that exhaust their gas content quickly (Lanfranchi \& Matteucci 2003). The low star formation rate, and thus slow metal enrichment, in dSph galaxies results in their stellar members possessing elemental abundance ratios similar to more metal-rich Galactic stars. Hence, the metal-poor $[\alpha / \mathrm{Fe}]$ values in dSphs would be lower than a Galactic halo star of the same metallicity. The contribution of $s$-process nuclei, produced primarily in long-lived low-to-intermediate mass stars, does not become important until [Fe/H] -1.0 dex in Milky Way Qian \& Wasserburg 2001; Travaglio et al. 2004a). However, the $s$-process production may become significant at lower $[\mathrm{Fe} / \mathrm{H}] \sim-1.7$ dex in dSph galaxies due to the slower metallicity increase (Venn et al. 2004; Lanfranchi et al. 2008). These expectations are seemingly confirmed by a growing body of dSph abundance data seen in, e.g., Figures 3, 5, 6, 7, 9, and 16 of Cohen \& Huang (2009); Figures 10 and 14 of (Letarte et al. 2010); and in Figures 11, 13, and 14 of the outstanding review of Tolstoy, Hill \& Tosi (2009).

Stephens \& Boesgaard (2002) used high-resolution and high S/N spectroscopy to determine abundances in some 50 halo field stars with $-3.6 \leq[\mathrm{Fe} / \mathrm{H}] \leq-0.7$ with peculiar kinematics or distinctive orbital properties. This sample exhibits a real dispersion in $[\alpha / \mathrm{Fe}]$, and Stephens \& Boesgaard (2002) find that their outer halo stars evince lower $[\alpha / \mathrm{Fe}]$ ratios than inner halo stars of the same metallicity. Despite their kinematic-based selection criteria, these authors were unable to identify any new low- $\alpha$ stars and concluded that their sample did not harbor the chemical hallmarks of an accreted extragalactic population.

Fulbright (2002) examined the abundances and kinematics of $\sim 70$ halo field stars with $-2 \leq[\mathrm{Fe} / \mathrm{H}] \leq-$ 1 in the Hipparcos catalog. A chemo-kinetic relation is manifest in these stars via the correlation between a variety of $[\mathrm{X} / \mathrm{Fe}]$ ratios (including $\mathrm{X}=\mathrm{Mg}, \mathrm{Si}$, and $\mathrm{Ca}$ ) and 3 -d space velocity. In their analysis of 94 metal-poor dwarfs, Nissen \& Schuster (2010) find their low $[\alpha / \mathrm{Fe}]$ stars show distinctive kinematics, which they interpret as a result of accretion from dwarf galaxies. However, Fulbright (2002) have called attention to differences in the abundance ratios of Galactic low- $\alpha$ stars and those measured in situ in local dwarf galaxies, suggesting the former do not have their origin in the latter.

An alternative interpretation of low- $\alpha$ halo stars is provided by Shigevama \& Tsujimoto (2003), who posit photospheric pollution due to accretion of iron-rich planetary material that increases the surface Fe abundance relative to the $\alpha$-elements. Smith et al. (2001) have noted the existence of Galactic disk exoplanet host stars that demonstrate increasing abundance $[\mathrm{X} / \mathrm{H}]$ with increasing condensation temperature $T_{\mathrm{C}}(\mathrm{X})$, and suggest that this correlation is consistent with photospheric accretion of (presumably once circumstellar) refractory material that leads to an abundance- $T_{\mathrm{C}}$ trend similar to that seen in chondritic solar system material (e.g., Figure 1a of Yin 2005). Photospheric $T_{\mathrm{C}}(\mathrm{X})$-dependent abundance trends are now commonly utilized as signatures of planet formation (e.g., Ramirez et al. 2010; Schuler et al. 2011a, b; ; Liu et al. 2014)

Chen \& Zhao (2006) called attention to correlations between $[\mathrm{X} / \mathrm{Fe}]$ values and $T_{\mathrm{C}}(\mathrm{X})$ in the low- $\alpha$ halo stars HD 134439 and 134440. They propose that such correlations originate from 
formation in a (chemically fractionated, refractory-rich) "dusty environment" in a dSph. Indeed, the apparent correlation they identify is a qualitative mirror image of the (gas-phase) ISM depletion versus $T_{\mathrm{C}}$ trend (e.g., Figure $1 \mathrm{~b}$ of Yin 2005).

Motivated by the various suggested origins of abundance patterns in low- $\alpha$ Galactic halo stars, we continue the delineation of abundances in the two metal-poor $([\mathrm{Fe} / \mathrm{H}] \sim-1.50)$ low- $\alpha$ halo field stars HD 134439 and 134440 (King 1997a). These two main sequence dwarfs are a weakly bound, high proper motion pair with a common origin evidenced by their shared kinematics and chemical abundances. We use newly-derived and existing literature abundances to explore the origin of these stars' low $[\alpha / \mathrm{Fe}]$. In particular, we present abundances for the light element Be, the volatile $\mathrm{CNO}$ elements, and the $n$-capture elements $\mathrm{Ag}$ and $\mathrm{Eu}$. We investigate whether there exists a relationship between elemental abundances and condensation temperature in these stars and, if so, its interpretation. Our central aim is to determine whether these 2 stars (and perhaps the Galactic population of low- $\alpha$ stars as a whole) are best understood in the context of star formation environment, circumstellar evolution, stellar evolution, extragalactic chemical evolution, or some combination of these.

\section{DATA AND ANALYSIS}

\subsection{Observations}

High-resolution $(R=49,000)$ spectroscopy of HD 134439 and 134440 was obtained on UT 19 June 2006 using the HIRES spectrograph on the W.M. Keck I 10-m telescope (Table 1). The useful wavelength coverage of the echelle spectra extends from 3020 to $5800 \AA$. Standard echelle reductions (debiasing, flat-fielding, order tracing and extraction, and wavelength calibration) were carried out. Certain regions of spectra overlapped in two adjacent orders and were coadded to increase $\mathrm{S} / \mathrm{N}$ ratio. The final average $\mathrm{S} / \mathrm{N}$ values near the $\mathrm{CH}, \mathrm{NH}$, and $\mathrm{OH}$ features of interest here are listed in Table 1. This per-pixel $\mathrm{S} / \mathrm{N}$ ratio is calculated from Poisson statistics in the pseudo-continuum near the features.

\subsection{Basic Physical Parameters}

The metallicity $([\mathrm{Fe} / \mathrm{H}])$, surface temperature $\left(\mathrm{T}_{\text {eff }}\right)$, and microturbulent velocity $(\xi)$ of HD 134439 and 134440 and their uncertainties are taken from King (1997a) and are listed in Table 2. Surface gravities taken from a 12 Gyr Yonsei-Yale $\left(\mathrm{Y}^{2}\right)$ isochrone (Demarque et al. 2004) with $[\alpha / \mathrm{Fe}]=0,[\mathrm{Fe} / \mathrm{H}]=-1.50$, and the Lejeune et al. (1998) color table are $\log g=4.77$ and 4.79 for

HD $134439\left(\mathrm{~T}_{\text {eff }}=5000 \mathrm{~K}\right)$ and HD $134440\left(\mathrm{~T}_{\text {eff }}=4785 \mathrm{~K}\right)$ respectively. Alternatively, $\log g$ can be calculated according to

$$
\log \frac{g}{g_{\odot}}=\log \frac{\mathrm{M}}{\mathrm{M}_{\odot}}+4 \log \frac{\mathrm{T}_{\text {eff }}}{\mathrm{T}_{\text {eff }, \odot}}+0.4 \mathrm{~V}_{\circ}+0.4 B C+2 \log \pi+0.12
$$


where $\mathrm{M}$ is the mass, $\mathrm{V}_{\circ}$ is the apparent magnitude, and $\pi$ is the parallax in arcsec; these values are given in Table 1 . The bolometric correction $(B C)$ is given by

$$
B C=\mathrm{M}_{b o l, \odot}-\mathrm{M}_{\mathrm{V}}-2.5 \log \frac{\mathrm{L}}{\mathrm{L}_{\odot}}
$$

We take $\mathrm{M}_{b o l, \odot}=4.71$ (from the Lejeune et al. 1998 color table according to Yi et al. 2001), the Hipparcos-based $\mathrm{M}_{\mathrm{V}}$ values from Table 1, and $\log \frac{\mathrm{L}}{\mathrm{L} \odot}$ values of -0.829 and -0.931 (for HD 134439 and 134440, respectively) from the $\mathrm{Y}^{2}$ isochrone; equation 2 then yields bolometric corrections of -0.29 and $-0.35 \mathrm{mag}$ for HD 134439 and 134440, respectively. Equation 1 with $\log g_{\odot}=4.44$ dex and $\mathrm{T}_{\text {eff, } \odot}=5770 \mathrm{~K}$ then yields physical gravities of $\log g=4.61$ and 4.63 for HD 134439 and 134440, respectively. Averaging the isochrone-based and parallax-based physical $\log g$ values, we obtain $\log g=4.69$ and 4.71 for HD 134439 and 134440; the differences between the isochrone and physical gravities around these means suggest an uncertainty at the \pm 0.10 dex level.

\subsection{Abundance Analysis}

The new abundances presented here are determined using Kurucz model atmospheres and an updated version of the LTE spectrum synthesis package MOOG package (Sneden 1973) that includes updated b-f opacity data relevant for the near-UV syntheses. The line list for the $3167.17 \AA$ $\mathrm{OH}$ line region is that compiled by Schuler et al. (2006). Other line lists used here are compiled from Kuruct 1 atomic and molecular lines (Kurucz 1995), the Vienna Atomic Line Database (Kupka et al. 2000), CH molecular lines from Jorgensen et al. (1996), and molecular lines simulated by LIFBASE (Luque \& Crosley 1999). CH, NH and $\mathrm{OH}$ diatomic dissociation energies adopted in the line lists are $3.47,3.45$, and $4.39 \mathrm{eV}$, respectively. Oscillator strengths ( $g f$-values) were adjusted to produce solar syntheses matching the Kurucz solar flux atlas (Kurucz 2005). The line lists are provided in Tables 3-7, which include wavelengths, species identifications, lower excitation potentials, and oscillator strengths.

Synthetic solar flux spectra were generated with elemental abundances from Anders \& Grevesse (1989) except for C, N, and O. The solar C and N abundances are from Asplund et al. (2005), while the solar $\mathrm{O}$ abundance is from Allende Prieto et al. (2001). We note that these adopted abundances not only set the $g f$ values used in deriving the stellar abundances, but are also subsequently used to solar-normalize those stellar abundances; our stellar abundances in the form $[\mathrm{X} / \mathrm{H}]$ are thus not dependent on the adopted solar CNO values. Elemental abundances from previous analyses (see $\S 3)$ were manual inputs in the stellar syntheses; abundances of other elements (except Be, C, N, $\mathrm{O}$, and $\mathrm{Ag}$, whose abundances are determined here) were scaled to the solar values according to the $[\mathrm{Fe} / \mathrm{H}]$ value taken here ( -1.47 dex and -1.53 dex for HD 134439 and 134440, respectively). A Gaussian smoothing factor, which accounts for instrumental and any modest macroturbulent

\footnotetext{
${ }^{1}$ http://kurucz.cfa.harvard.edu/linelists.html
} 
broadening, was obtained from Doppler-corrected FWHM measurements of weak clean observed spectral lines at redder wavelengths (4000-5800 $\AA$ ), and then applied to the synthetic spectra.

The stellar spectra were first normalized relative to the pseudo-continuum with SPECTRE (Fitzpatrick \& Sneden 1987). Small wavelength shifts and flux rescalings were applied to improve the match with the synthetic spectra generated by MOOG. We determined best-fit abundances via $\chi^{2}$ minimization of the synthesized and normalized observed spectra over regions centered on features that were identified as sufficiently strong and clean to allow good abundance measurements; we discarded those features that were too saturated or severely blended. After initial CNO abundances were measured, they were used as inputs for new syntheses in order to enforce molecular equilibrium. The abundances reported in Tables 8, 9, and10 are final measurements after achieving molecular equilibrium.

\subsection{Carbon Abundances}

Seven $\mathrm{CH}$ lines were used in the $\lambda 4322-4326 \mathrm{G}$-band region to measure the $\mathrm{C}$ abundance (see Figure 1). The abundances are measured by applying $\chi^{2}$ measures to individual features, instead of the entire band. Final best fit abundances are listed in Table 8. The mean carbon abundances found for HD 134439 and 134440 are $\log N(\mathrm{C})_{39}=6.49$ and $\log N(\mathrm{C})_{40}=6.162$, yielding $[\mathrm{C} / \mathrm{H}]_{39}=-1.90$ for $\mathrm{HD} 134439$ and $[\mathrm{C} / \mathrm{H}]_{40}=-2.23$ for HD 134440 after self-consistently employing the solar $\mathrm{C}$ abundance used in our line list calibration.

\subsection{Nitrogen Abundances}

Four NH lines in the $\lambda 3326-3332$ region were used for the calculation of the $\mathrm{N}$ abundance (see Figure 2). Although there are more than four NH features in the spectral region, we found them too strong or blended relative to the selected lines. The abundances given by the best fit between the synthetic and observed spectra for individual NH lines are listed in Table 9. The resulting average nitrogen abundances are $\log N(\mathrm{~N})_{39}=5.83$ and $\log N(\mathrm{~N})_{40}=5.68$. The ratios relative to the Sun are $[\mathrm{N} / \mathrm{H}]_{39}=-1.95$ and $[\mathrm{N} / \mathrm{H}]_{40}=-2.10$.

\subsection{Oxygen Abundances}

The $\mathrm{O}$ abundance is measured with seven near-UV electronic $\mathrm{OH}$ features in the range 3129$3168 \AA$ (Figure 3). Line-by-line results are listed in Table 10, Final average oxygen abundances are

\footnotetext{
${ }^{2}$ The "39" and "40" subscripts designate HD 134439 and 1344404, respectively; logarithmic number abundances are on the traditional scale where $\log N(\mathrm{H}) \equiv 12$.
} 
$\log N(\mathrm{O})_{39}=7.02$ and $\log N(\mathrm{O})_{40}=6.83$. The $\mathrm{O}$ abundances relative to the Sun are $[\mathrm{O} / \mathrm{H}]_{39}=$ -1.67 and $[\mathrm{O} / \mathrm{H}]_{40}=-1.86$.

Because the final CNO abundances are normalized with respect to the Sun self-consistently, they are independent of adopted $g f$-values and solar abundances. Utilizing, for example, solar abundances different from those employed here would require modifications of the $g f$-values in the line lists to match the solar atlas. Resulting stellar syntheses would produce elemental abundances that are adjusted accordingly. These absolute abundances, however, would then be normalized by an equivalently different adopted solar abundance. Hence, abundances of the elements that are consistently normalized to the Sun will be unaffected by the solar values assumed in the line list calibration.

\subsection{Abundance Sensitivities and Uncertainties}

Uncertainties in our abundances arise from uncertainties in effective temperature, surface gravity, microturbulent velocity, the (pseudo-)continuum setting, and measurement uncertainty. Uncertainties in the $[\mathrm{CNO} / \mathrm{Fe}]$ ratios due to uncertainties in the stellar parameters are derived from the parameter sensitivities, which are listed in columns 2, 3, and 4 of Table 11, The sensitivities of $[\mathrm{Fe} / \mathrm{H}]$, which are folded in to the listed $[\mathrm{X} / \mathrm{Fe}]$ sensitivities, are taken from Table 3 of King (1997a), who uses the same model atmospheres and is the source of the $[\mathrm{Fe} / \mathrm{H}]$ values and adopted parameters.

While the observed spectra's (pseudo-)continuum shape and absolute level are difficult to determine in isolation, the syntheses provide guidance. Repeating comparison with syntheses after altering the continuum level of the observed spectra by plausible amounts, we believe the $1 \sigma$ level uncertainties in the average abundance of $\mathrm{C}, \mathrm{N}$ and $\mathrm{O}$ are 0.04 dex to 0.06 dex. The line-by-line spread, parameterized as a statistical uncertainty in the mean $\sigma_{\mu}$, of the $\mathrm{C}$ or $\mathrm{N}$ or $\mathrm{O}$ abundances provides an estimate of the measurement uncertainties. These values for the $7 \mathrm{CH}$ lines, $4 \mathrm{NH}$ lines

and $7 \mathrm{OH}$ lines are combined in quadrature with the uncertainty in the mean for $[\mathrm{Fe} / \mathrm{H}](\mathrm{King}$ 1997a), yielding the statistical uncertainties in the mean $[\mathrm{X} / \mathrm{Fe}]$ given in the penultimate column of Table 11.

The total uncertainties in the $[\mathrm{CNO} / \mathrm{Fe}[$ ratios are found by adding the parameter-based, continuum-based, and measurement uncertainties in quadrature, and are listed in listed in the final column of Table 11. The major contribution to the final uncertainties originates from uncertainties in $\mathrm{T}_{\text {eff }}$; our elemental abundances are less sensitive to other quantities, especially $\xi$. The mean [CNO/Fe] values in HD 134439 versus HD 134440 differ by 0.09-0.27 dex. The difference between the HD 134439 and $134440 T_{\text {eff }}$ values according to the newer infrared flux method results of Casagrande et al. (2010) is $88 \mathrm{~K}$ lower than that associated with our adopted values. This would diminish the $[\mathrm{CNO} / \mathrm{Fe}]$ differences by 0.05-0.09 dex, bringing the $[\mathrm{NO} / \mathrm{Fe}]$ ratios into near-perfect agreement and leaving a 0.22 dex difference in $[\mathrm{C} / \mathrm{Fe}]$. Regardless, our $\mathrm{O}$ and $\mathrm{N}$ ratios in the two 
stars differ at the $\leq 1 \sigma$ level, and the 0.27 dex difference in the $\mathrm{C}$ ratio is at the $2 \sigma$ level; a difference of this latter size in one of the 22 elemental ratios collected in Table 7 is expected in a statistical sense. The modest CNO differences between the two stars do not affect the salient result that $[\mathrm{CNO} / \mathrm{Fe}]$ are all markedly low compared to the Galactic halo field.

Regarding possible systematic errors, the referee reminds us that the CNO abundances derived from molecular features depend sensitively on the $T-\tau$ structure of the upper layers of the model atmospheres. Computations of 3D hydrodynamic model atmospheres suggests that the adequacy of the $T-\tau$ structure of $1 \mathrm{~d}$ hydrostatic model atmospheres declines with metallicity (compare, e.g., Figures 1 and 2 of Asplund 2005). Any metallicity-dependent component of this difference would not be removed by a differential analysis with respect to the Sun. One way to mitigate the impact of such possible effects is to compare our abundances with those in similar metallicity Galactic halo stars that are also derived from molecular features; we do this in $§ 3.2$.

\section{RESULTS AND DISCUSSION}

We group and list 22 elements with abundance determinations in HD 134439/440 in the first two columns of Table 12. Columns 3 and 4 give the [X/Fe] values for HD 134439 and 134440, respectively; $\mathrm{CNO}$ and $\mathrm{Ag}$ abundances are those derived in this study, Fe is taken from King (1997a), and the remainder are from Chen \& Zhao (2006). Column 5 contains the average $[\mathrm{X} / \mathrm{Fe}$ and estimated uncertainty. Condensation temperatures for the elements (from Table 8 of Lodders 2003) are listed in the final column.

\subsection{Abundances and $\mathbf{T}_{\mathrm{C}}$}

We plot the average HD 134439/440 abundance ratio versus condensation temperature in Figure 4. The CNO abundance ratios (at low $\mathrm{T}_{\mathrm{c}}$ ) are comparatively lower than those of the majority of refractory elements at high $\mathrm{T}_{\mathrm{c}}$. The size of the Spearman's rank coefficient (0.422), which measures the correlation between the abundances and $T_{c}$ via the monotonicity of the rankings of the two variables with no underlying assumptions concerning their parent distributions (Wall \& Jenkins 2003 ), indicates only a $5 \%$ probability that the observed correlation could occur by chance in an uncorrelated sample-not inconsistent with claims of chemical pollution via planetesimal/refractory accretion (Shigevama \& Tsujimoto 2003).

The mean difference in abundance ratios (HD 134439 - HD 134440;) from Table 12 is 0.02 dex with scatter $( \pm 0.08 \mathrm{dex})$ that is consistent with uncertainties. Given a) the two stars' convection zone depths that differ by $\geq 15 \%$ based upon our $\mathrm{T}_{\text {eff }}$ and the model estimates from Figure 2 of Richard. Michaud \& Richer (2002), and b) possible differences in the mass/composition of material putatively accreted by each star, remarkable fine-tuning would have been required for them to now exhibit indistinguishable post-accretion abundance patterns. We are thus led to examine other 
interpretations of the $[\mathrm{X} / \mathrm{Fe}]$ verus $\mathrm{T}_{\mathrm{c}}$ relationship.

\subsection{Comparisons with the Galactic Halo}

One such interpretation is that the $[\mathrm{X} / \mathrm{Fe}]-\mathrm{T}_{\mathrm{c}}$ correlation is simply an unremarkable result of Galactic chemical evolution. We explore this possibility by comparing HD 134439 and 134440 to Galactic halo stars of similar $[\mathrm{Fe} / \mathrm{H}]$, adopting typical $[\mathrm{X} / \mathrm{Fe}]$ values of halo stars with $[\mathrm{Fe} / \mathrm{H}] \sim-1.50$ using the observational data from Kobavashi et al. (2006), Zhang et al. (2009), and Nissen \& Schuster (2010) (for their high- $\alpha$ stars) with a few exceptions. First, we believe the high $[\mathrm{N} / \mathrm{Fe}]$ values at $[\mathrm{Fe} / \mathrm{H}] \sim-1.50$ shown in Kobayashi et al. (2006) are due to mixing in evolved giants. The nearUV NH-based $[\mathrm{N} / \mathrm{Fe}]$ values in unmixed or unevolved stars from the studies of Spite et al. (2005) and Israelian et al. (2004) consistently indicate that $[\mathrm{N} / \mathrm{Fe}]=0.0$ at $[\mathrm{Fe} / \mathrm{H}]=-1.5$. The primary nucleosynthetic behavior of $\mathrm{N}$ this result implies has long been suspected-grave uncertainties in previous N abundance determinations not withstanding (e.g., Wheeler et al. 1989 and Prantzos 2003). A value of $[\mathrm{N} / \mathrm{Fe}]=0.0$ at $[\mathrm{Fe} / \mathrm{H}]=-1.5$ is also in excellent agreement with a metal-poor extension of the CN-based [N/Fe] ratios in more metal-rich giants from Carretta et al. (2000) that are presumably immune from the effects of CN-cycling.

Second, we adopt the OH-based oxygen ratios in metal-poor field stars of Boesgaard et al. (1999, 2011) to compare with our molecular-based results in HD 134439/440. Third, we adopt the CH-based carbon ratios in metal-poor field stars from Tomkin et al. (1992), Gratton et al. (2000), Simmerer et al. (2004), and Lai et al. (2007) to compare with our molecular-based results. Fourth, we adopt $\mathrm{Al}$ and $\mathrm{K}$ data compiled by Timmes et al. (1995, and references therein). Fifth, we adopt the Galactic Y and Ba data compiled by Travaglio et al. (2004a). Finally, Galactic Ag data is taken from Crawford et al. (1998).

The mean Galactic halo abundance ratios at $[\mathrm{Fe} / \mathrm{H}] \sim-1.5$ have a negligible correlation with $T_{\mathrm{C}}$ (upper panel of Figure 5). The contrast between the mean Galactic halo field and HD $134439 / 134440$ is seen in the lower panel of Figure 5. the differential abundance ratios (our stars halo) demonstrate a correlation with $T_{\mathrm{C}}$ at the $\sim 99.5 \%$ confidence level. This is largely driven by CNO: the Spearman-based correlation excluding CNO is significant at only the $\sim 85 \%$ confidence level.

Given the multiplicity of nucleosynthetic sites for CNO production and uncertainties in our understanding of non-standard stellar mixing, winds, etc, it is possible that the $[\mathrm{X} / \mathrm{Fe}]-T_{\mathrm{C}}$ correlation in HD 134439/440 is simply the consequence of integrated stochastic effects of their chemical evolution. However, the unusual $V_{\mathrm{LSR}}, R_{\text {apo }}$, and $Z_{\max }$ values of our 2 stars suggest their relevant preceding chemical evolution history did not transpire in the Galaxy (Carnev et al. 1996). Thus, we explore alternate explanations of the abundance correlation and the low- $\alpha$ phenomenon. 


\subsection{1. $\mathrm{O}, \mathrm{Mg}, \mathrm{Al}$, and Proton-Capture}

There are interesting similarities between the HD 134439/440 abundance patterns and those of Galactic globular cluster red giants, for which considerable evidence of proton-capture nucleosynthesis has been observed (Kraft 1994; Pilachowski et al. 1996; Kraft et al. 1997, 1998). Deep mixing-in situ or in previous stellar generations having enriched the cluster gas-has apparently enhanced some cluster giant $\mathrm{N}, \mathrm{Na}$, and $\mathrm{Al}$ abundances by conversion of $\mathrm{C} \mathrm{O}, \mathrm{Ne}$, and $\mathrm{Mg}$ nuclei through proton-capture. As a result, one can observe low $[\mathrm{O} / \mathrm{Fe}]$ and $[\mathrm{Mg} / \mathrm{Al}]$ ratios for many cluster giants compared to enhanced ratios observed in halo field giants and those expected from Type II SNe nucleosynthesis. The mean Galactic halo field data (Figure 5 ) evince [O/Fe] 0.57 and $[\mathrm{Mg} / \mathrm{Al}] \sim 0.70$ at $[\mathrm{Fe} / \mathrm{H}] \sim-1.50$. The corresponding ratios for HD 134439/440 (Table 12) are 0.83 and 0.60 dex lower, and more similar to the ratios exhibited by many globular cluster giants.

The Na and N abundances of HD 134439/440, however, exclude proton-capture as the origin of this similarity. Cluster giants with $[\mathrm{O} / \mathrm{Fe}] \sim-0.25$ (like HD 134439/440) demonstrate $[\mathrm{Na} / \mathrm{Fe}] \sim 0.40$ (see Figure 4 of Kraft et al. 1998); the mean [Na/Fe] -0.48 for HD 134439/440 is not similarly enhanced. In fact, the HD 134439/440 Na abundance is low compared to the mean halo field data $([\mathrm{Na} / \mathrm{Fe}] \sim-0.16)$. Similarly, our $\mathrm{N}$ abundance is also lower than the proton-capture processed material in cluster giants or in the mean halo field. The deep mixing models shown in Figures 1-3 of Langer \& Hoffman (1995) conserve the sum of $\mathrm{C}+\mathrm{N}+\mathrm{O}$; our Figure 5 indicates that this sum in HD 134439/134440 is $\leq 20 \%$ (by number) of the sum for halo field stars at $[\mathrm{Fe} / \mathrm{H}] \sim-1.5$. Given these key comparisons, we reject proton-processing as the agent responsible for our stars' anomalous abundances with respect to the halo field.

\subsection{Extragalactic Origins: Dust}

Because Galactic halo field stars showing low $[\alpha / \mathrm{Fe}]$ and $[\mathrm{Na} / \mathrm{Fe}]$ in the studies of Nissen \& Schuster (1997) and Nissen \& Schuster (2010) demonstrate distinctive kinematics, these authors hypothesize an extragalactic origin (e.g., dwarf galaxies) for such chemically anomalous stars. HD 134439/440 also exhibit low $[\alpha / \mathrm{Fe}]$ and $[\mathrm{Na} / \mathrm{Fe}]$ as well as large $\mathrm{R}_{\max }(\sim 43 \mathrm{kpc})$, and a dSph origin has previously been posited for these two stars (King 1997a; Chen \& Zhao 2006). The latter authors suggest formation in a high dust-to-gas environment, which might explain the HD 134439/440 abundance ratio- $T_{\mathrm{C}}$ correlation and low $\alpha$-element abundance ratio, as an alternative to the planetesimal accretion mechanism proposed to explain these abundance data (Shigeyama \& Tsujimoto 2003). In the Chen \& Zhao (2006) scenario, dust from ancestral stellar generations is enriched in (high $T_{\mathrm{c}}$ ) refractory elements relative to (low $T_{\mathrm{c}}$ ) volatiles-consistent with the opposite pattern evinced by gas-phase ISM results (Field 1974); this dusty pollutant, combined with natal pre-stellar material, would end up back in the HD 134439/134440 photosphere we observe today, creating a correlation of abundance with $T_{\mathrm{c}}$.

We show the HD 134439/440 abundance ratios and those for dSph red giants in Figures [6] and 7 . 
The abundances for Sagittarius (Sgr) are obtained from Bonifacio et al. (2000, 2004), Monaco et al. (2005), and Smecker-Hane \& McWilliam (1999). We use results from Shetrone et al. (2001, 2003) for giants in Draco (Dra), Ursa Minor (UMi), Sextans (Sex), Sculptor (Scl), Fornax (For), Carina (Car), and Leo I. We have also adopted the Geisler et al. (2005) abundance measurements for four red giants in Scl. Due to the lack or sparseness of dSph C, N, Eu, and Ag measurements (particularly at $[\mathrm{Fe} / \mathrm{H}] \sim-1.5$ ), panels for these elements are omitted. A datapoint corresponding to the average HD 134439/440 abundance ratio is included in each panel of Figures 6 and 7, but is not easily discerned within the dense dSph scatter. Indeed, the HD 134439/440 abundance ratios fit within the manifest continuum of in situ dSph abundances that result from their individual histories (Lanfranchi et al. 2008).

Direct evidence against an unusually dusty pre-stellar environment for HD 134439/440 is, however, found in their Be abundances. We determined these via synthesis of the $\lambda 3130.4,3131.1$ Be II doublet as accomplished for halo field stars in Boesgaard et al. (2011). While the ionized doublet of this trace element in our metal-poor high-gravity stars is vanishingly weak, we set an upper limit of $\log N(\mathrm{Be})=-0.7$ (on the usual scale where $\log N(\mathrm{H})=12$.) in HD 134439. This upper limit is $\sim 0.7$ dex lower than the abundance of Galactic halo field stars of similar $[\mathrm{Fe} / \mathrm{H}]$ seen in Figure 9 of Boesgaard et al. (2011). A deficiency of this high- $T_{\mathrm{C}}(1450 \mathrm{~K})$ element is inconsistent with the enhancement expected if our star was formed in a refractory-rich environment or had accreted refractory-rich material.

Instead, the upper limit is consistent with the Be abundances of Galactic halo field stars of similar $[\mathrm{O} / \mathrm{H}]$ (Figure 11 of Boesgaard et al. 2011). That the HD 134439 Be abundance appears anomalously low relative to Galactic halo field stars when referenced to Fe, but unremarkable when referenced to $\mathrm{O}$ is qualitatively in accord with: a) the spallation origin of Be (Reeves. Fowler \& Hoyle 1970) linking its nucleosynthetic history to that of O, and b) the well-known differences between $[\mathrm{O} / \mathrm{Fe}]$ (and other $[\alpha / \mathrm{Fe}]$ ratios) in the Galactic halo and dSphs. For example, Figure 8 from King (2000) and our Figure 6 indicates that $[\mathrm{O} / \mathrm{Fe}]$ is $\geq 0.4$ dex higher at $[\mathrm{Fe} / \mathrm{H}]=-1.5$ in the Galactic halo; this difference is confirmed by the Galactic and dSph $[\mathrm{Mg} / \mathrm{Fe}]$ ratios seen in, e.g., Figure 1 of Tsuijimoto (2006) or Figure 11 of Tolstov, Hill \& Tosi (2009)

The referee notes that Galactic halo field Be abundances are determined in stars $\sim 500-1000$ $\mathrm{K}$ warmer than HD 134439. This raises the possibility that the initial Be abundance of HD 134439 might have been larger than that in hotter Galactic field stars of similar $[\mathrm{O} / \mathrm{H}]$, but now is lower due to greater stellar depletion in our cooler dwarf. Unfortunately, the Be- $T_{\text {eff }}$ trend in metal-poor stars that would trace the effects of differential depletion is not observationally defined. However, stellar models suggest that the difference in Be depletion in 5000 and $6000 \mathrm{~K}$ old metal-poor dwarfs is zero or in the opposite sense suggested (i.e., hotter model stars deplete slightly more Be). The $Z=0.001$ standard stellar model results in Table 3A of Pinsonneault. Delivannis \& Demarque (1992) suggest no depletion in old $5000 \mathrm{~K}$ or $6000 \mathrm{~K}$ stars. The rotational stellar models in Tables 4A-4C of Pinsonneault, Deliyannis \& Demarque (1992) suggest that Be depletion may be $\leq 0.2$ dex greater at $6000 \mathrm{~K}$ than at $5000 \mathrm{~K}$. Such a modest effect, if real, would only strengthen our conclusion 
about the difference in Be abundance between HD 134439 and the Galactic halo field.

We note that there is no evidence from the $\lambda 3131$ features of any Be in the cooler, less massive HD 134440. A real difference in our two stars' Be content may not be surprising. The temperature of the surface convection zone base, its temporal evolution, and thus the extent and length of Be destruction due to pre-main-sequence and main-sequence convective mixing, are highly sensitive functions of mass in the mass regime occupied by our stars (Bodenheimer 1966).

\subsection{Stochastic Nucleosynthetic Origins: Type II Contributions}

None of the preceding mechanisms-Galactic halo-like chemical evolution, (proto-)planetary accretion, formation in an unusually dusty environment, or formation from material enriched in stellar $p$-capture products-provides a plausible or convincing explanation of the $[\mathrm{X} / \mathrm{Fe}]-T_{\mathrm{C}}$ relation in HD 134439/440. We thus consider whether this relation is simply an illusory one stemming from these stars' unique chemical evolutionary history. Motivation and guidance in doing so is provided by Tolstoy et al. (2003), who analyze stellar abundances in four dSph galaxies and find $\alpha$-element abundance ratios suggesting negligible nucleosynthetic contributions from stars of progenitor mass $\geq 15-20 \mathrm{M}_{\odot}$. Tolstoy et al. (2003) note that such a mass function truncation may be only effective, and other possible explanations (e.g., star formation efficiency and metal-loss by winds) having the same observable consequence do exist. We explore the effects of mass function truncation here using the Type II yields of Kobayashi et al. (2006), and find that explosive nucleosynthesis in Type II SNe of low progenitor mass provides an attractive explanation for the abundance patterns of HD $134439 / 440$.

The $[\mathrm{O} / \mathrm{Fe}]$ ratio and those of the higher $T_{\mathrm{C}} \alpha$-elements $\mathrm{Mg}, \mathrm{Si}$, and $\mathrm{Ca}$ are lower in $\mathrm{HD}$ 134439/440 than in the mean Galactic halo (columns 5 and 6 of Table 12). The Galactic potassium abundance ratios used in Figure 5 exhibit behavior strongly reminiscent of traditional $\alpha$-elements; the markedly low $[\mathrm{K} / \mathrm{Fe}]$ ratio of our stars relative to those in the Galactic halo at similar $[\mathrm{Fe} / \mathrm{H}]$ (a difference of 0.4 dex as seen in Table 12) is thus a notable similarity. In sum, the HD 134439/440 $\alpha$-element ratio resembles the sub-Galactic halo values seen in dSph stars. We now seek additional evidence in the HD 134439/440 abundance patterns that might support the idea of their origin in a dSph-like environment whose prior chemical evolution was dominated by the effects of a small number of discrete nucleosynthetic events characterized by mass function truncation.

Such supporting evidence can be found in these stars' intra- $\alpha$ element ratios. In particular, $[\mathrm{O} / \mathrm{Fe}]$ is $0.15-0.45$ dex lower than ratios of other $\alpha$-elements; we suggest this has little to do with $T_{\mathrm{c}}$-related behavior per se. Instead, we note that the $[\mathrm{O} / \mathrm{Fe}]$ yields in, e.g., $Z=0$ Type II models of Kobavashi et al. (2006) are steep functions of mass-some 0.60 dex lower for progenitors of 14 $\mathrm{M}_{\odot}$ than for $20 \mathrm{M}_{\odot}$. In contrast, the $[\mathrm{Mg} / \mathrm{Fe}]$ and $[\mathrm{Si} / \mathrm{Fe}]$ yields are much shallower functions of mass; their yields change by $\leq 0.20$ dex over the same progenitor mass range. Formation from material enriched by Type II SNe biased towards low progenitor mass is qualitatively consistent 
with low $[\mathrm{O} / \mathrm{Fe}]$ compared to $[\mathrm{Mg}, \mathrm{Si} / \mathrm{Fe}]$ in $\mathrm{HD}$ 134439/440. Indeed, for progenitor mass of $14 \mathrm{M}_{\odot}$, the $[\mathrm{Mg}, \mathrm{Si} / \mathrm{Fe}]$ yields are some 0.30 dex larger than for $[\mathrm{O} / \mathrm{Fe}]-$ a difference in excellent agreement with observed values.

The HD 134439/440 [Na/Fe] ratio is anomalously low as well, lying some 0.3 dex below the Galactic halo mean (Table 12). As is the case for [O/Fe], the Kobavashi et al. (2006) model [Na/Fe] yields also drop precipitously over the 20 to $14 \mathrm{M}_{\odot}$ progenitor mass range. In Figure 8, we compare the observed abundance ratios in HD 134439/440 with those of the Kobavashi et al. (2006) yields for a $14 \mathrm{M}_{\odot}$ progenitor. The observed $[\mathrm{Na} / \mathrm{Fe}]$ is well-matched by the low mass progenitor yield. The distinctly sub-solar ratio $[\mathrm{Na} / \mathrm{Fe}]$ ratio $(\sim-0.50)$ of HD $134439 / 440$ is thus easily explained by our proposed nucleosynthetic origin without recourse to $T_{\mathrm{c}}$-related effects.

The $[\mathrm{C} / \mathrm{Fe}]$ and $[\mathrm{N} / \mathrm{Fe}]$ values of $\mathrm{HD} 134439 / 134440$ are also low compared to the Galactic halo (Table 12); the differences are even a factor of two lower than for $[\mathrm{O} / \mathrm{Fe}]$. This too needs have little to do with $T_{\mathrm{c}}$-related effects. Chemical evolution models of dSph galaxies predict $[\mathrm{N} / \mathrm{O}]$ and [C/O] ratios as low as -1 (Figure 13 of Carigi \& Hernandez 2008), which easily accommodates the values of $\sim-0.30$ in our stars if they formed in such an environment.

Figure 8 shows that the $[\mathrm{Zn}, \mathrm{Ni}, \mathrm{Co} / \mathrm{Fe}]$ values of our stars agree with the $\sim 14 \mathrm{M}_{\odot}$ progenitor mass yields of Kobavashi et al. The observed [Al, Cr/Fe] ratios are seen to be virtually identical to these $14 \mathrm{M}_{\odot}$ progenitor yields. The value $[\mathrm{Cu} / \mathrm{Fe}] \sim-0.70$ for our 2 stars also need not be associated with $\mathrm{T}_{\mathrm{C}}$-related effects inasmuch as it is not significantly different than the ratio observed

in Galactic halo or dSph stars (Figures 5 and 7). As noted by Timmes et al. (1995), Galactic halo $\mathrm{Cu}$ data are consistent with the Type II yields of Woosley \& Weaver (1995), suggesting $\mathrm{Cu}$ production is dominated by explosive nucleosynthesis and not production in Type Ia supernovae or low-to-intermediate mass stars.

A couple puzzles remain. First, the observed $[\mathrm{Mn} / \mathrm{Fe}]$ value is 0.30 dex larger than the Kobayashi et al. (2006) $[\mathrm{Mn} / \mathrm{Fe}]$ yield at $14 \mathrm{M}_{\odot}$; however, it's interesting to note that the observed value is not anomalous with respect to dSph stars in this regard (Figure 7). Second, as already noted by Chen \& Zhao (2006), our stars' [Ti, Ca/Fe] values are both larger than [Mg/Fe], which is consistent neither with the Kobavashi et al. (2006) [X/Fe] yields at low progenitor mass nor the dSph results of Venn et al. (2004) who find [Ti,Ca/Fe] ratios smaller than $[\mathrm{Mg} / \mathrm{Fe}]$. We return to the $[\mathrm{Ti}, \mathrm{Ca} / \mathrm{Fe}]$ ratios in $\S 3.6$.

\subsection{Type Ia Contributions}

The preceding comparisons suggest that the abundance ratios of HD 134439/440 are dominated by nucleosynethic products of low mass progenitor Type II SNe. We find no observable evidence of Type Ia contributions in the abundance data. Such contributions are of possible interest in explaining the absolute levels of $[\mathrm{O} / \mathrm{Fe}]$ and $[\mathrm{Mn} / \mathrm{Fe}]$, which are some 0.30 dex lower and higher (respectively) than the Kobayashi et al. (2006) $14 \mathrm{M}_{\odot}$ progenitor yields; these differences 
are qualitatively consistent with lower and higher (respectively) ratios expected from Type Ia event yields.

We constructed simple Type II/Ia mixture models from the Kobavashi et al. (2006) Type II yields for a given progenitor mass and the Type Ia yields from Thielemann et al. (2003) as tabulated in Travaglio et al. (2004b). The Type Ia yield was weighted so as to reproduce the observed mean $[\mathrm{O} / \mathrm{Fe}]$ of our stars when combined with the Type II yields. Resulting $[\mathrm{X} / \mathrm{Fe}]$ yields from the Type II/Ia mixture were then computed. This process was then repeated using different Type II progenitor masses to produce multiple Type II/Ia mixture models. All such mixtures are unable to consistently reproduce the observed abundance ratios. We typically find that, simultaneously, the $[\mathrm{Ni} / \mathrm{Fe}]$ yields are $\geq 0.30$ dex larger than observed, $[\mathrm{Mn} / \mathrm{Fe}]$ becomes overenhanced by $\geq 0.25$ dex, $[\mathrm{Na} / \mathrm{Fe}]$ is $\geq 0.30$ lower than observed, and the predicted $[\mathrm{Ti} / \mathrm{Fe}]$ yield ratios become even lower compared to the data.

\subsection{Neutron-capture Elements}

\subsubsection{Heavy $n$-capture elements: $A g$, Eu, and $B a$}

While silver may seem an unconventional choice of element with which to explore $n$-capture nucleosynthesis, its great utility comes from observably strong transitions of neutral features in the near-UV. The observed $n$-capture transitions upon which most of our knowledge of Galactic and dSph abundance patterns are built are primarily singly ionized lines in low gravity red giants that can be vanishingly weak in our metal-poor cool dwarfs. We measured Ag abundances for HD 134439 and HD 134440 from synthesis of the $\lambda 3280$ and $\lambda 3382 \mathrm{Ag}$ I line regions. Line lists were compiled for the $\mathrm{Ag}$ line regions using VALD and assuming a solar $\mathrm{Ag}$ abundance of $\log N(\mathrm{Ag})_{\odot}=1.54$. We find $[\mathrm{Ag} / \mathrm{Fe}]=-0.19$ and -0.13 for HD 134439 and 134440 (Figure 9) with an uncertainty of 0.14 dex. Crawford et al. (1998) determined [Ag/Fe] in the metal-poor cool halo dwarf HD 103095, which has physical properties $\left(\mathrm{T}_{\text {eff }}=5007 \mathrm{~K}, \log g=4.65,[\mathrm{Fe} / \mathrm{H}]=-1.27\right.$ ) similar to HD 134439 , obtaining $[\mathrm{Ag} / \mathrm{Fe}]=+0.28 \pm 0.17$ dex from the same $\mathrm{Ag}$ I lines. The significantly lower $[\mathrm{Ag} / \mathrm{Fe}]$ value compared to that in HD 103095 can easily be seen by comparing the relative strengths of the neighboring Ag I and Fe I lines in Figure 9 with those of Figure 3 in Crawford et al. (1998).

We sought to compare [Eu/Fe] in HD $134439 / 134440$ and 103095 by synthesizing the $\lambda 4205$ Eu II lines. For HD 103095, we utilized a high S/N, R 60,000 spectrum obtained with the McDonald Observatory $2.7 \mathrm{~m}$ 2dCoude spectrograph and the stellar parameters adopted by King (1997b): $\mathrm{T}_{\text {eff }}=5050 \mathrm{~K},[\mathrm{Fe} / \mathrm{H}]=-1.31, \log g=4.5$, and $\xi=1.5 \mathrm{~km} / \mathrm{s}$; the McDonald spectrum does not allow for a direct comparison of $\mathrm{Ag}$ abundances because it does not extend to the atmospheric cutoff region near $3300 \AA$. The line profile at $4205.04 \AA$ appears blended in our stars and we encountered difficulties simultaneously and consistently fitting the wings of the line in the stars and the Sun (for which we assumed $\log N(\mathrm{Eu})_{\odot}=0.51$ in making $g f$-value adjustments). At present, we are only confident in establishing upper limits on [Eu/Fe] by fitting the $\lambda 4205.04$ line depth. This yields 
ratios of $[\mathrm{Eu} / \mathrm{Fe}] \lesssim+0.31$ for HD $134439, \lesssim+0.42$ for HD 134440 , and $\lesssim+0.70$ for HD 103095 . The suggestion that $[\mathrm{Eu} / \mathrm{Fe}]$ is $\sim 0.30-0.40$ dex larger in HD 103095 is confirmed in Figure 10, where we compare the observed spectra of HD 103095 (dotted line) with HD 134439 (solid line) over four spectral regions containing Eu II features; these features are consistently stronger relative to other metal features in HD 103095 compared to HD 134439.

Our Eu and $\mathrm{Ag}$ results and the Ba abundance given in Table 12 indicate that [Ba, $\mathrm{Ag}, \mathrm{Eu} / \mathrm{Fe}$ ] ratios are consistently lower in HD $134439 / 134440$ by $\sim 0.40$ dex compared to the mean Galactic halo field at $[\mathrm{Fe} / \mathrm{H}]=-1.5$. Marked deficiencies $(\geq 1 \mathrm{dex})$ in the heavy $n$-capture elements are seen in the low- $\alpha$ star $\mathrm{BD}+80245([\mathrm{Fe} / \mathrm{H}] \sim-2.05)$, which has $[\mathrm{Ba} / \mathrm{Fe}]=-1.87$ and -1.89 and $[\mathrm{Eu} / \mathrm{Fe}]=-1.04$ and -0.64 according to Fulbright (2000) and Ivans et al. (2003). Establishing the origin of these deficiencies requires examining lighter $n$-capture elements. In doing so, one must use care in dividing $n$-capture elements into traditional $s$ - and $r$-process categories. Canonical ascriptions are usually based on solar-system contributions, and may not hold in other contexts. Moreover, it has become clear that a distinct light element primary process (LEPP) process is required to explain $n$-capture abundances in the Galactic field (Travaglio et al. 2004a).

\subsubsection{The heavy-to-light n-capture abundance ratio}

As noted by Chen \& Zhao (2006), HD 134439/440 exhibit a peculiar $n$-capture abundance pattern: their mean $[\mathrm{Ba} / \mathrm{Y}] \sim-0.10$ is smaller than the value of +0.20 exhibited by Galactic halo stars of similar metallicity (Table 5$)$, and markedly lower than the mean value $(+0.60)$ of the significant and real scatter evinced by dSph stars (Shetrone et al. 2003; Venn et al. 2004; Tolstov, Hill \& Tosi 2009). Our stars are apparently deficient in heavy relative to light $n$-capture elements compared to the Galactic halo field and the majority of dSph systems studied to date.

Figures 5 and 10 of Travaglio et al. (2004a) indicate that $s$-contributions alone from AGB stars would result in $[\mathrm{Ba} / \mathrm{Y}]=+0.60$ to +0.70 at $[\mathrm{Fe} / \mathrm{H}]=-1.50$ in the Galaxy. Indeed, several authors (e.g., Venn et al. 2004; Pompeia et al. 2008; Letarte et al. 2010) invoke such contributions to explain the scattered but clearly supersolar $[\mathrm{Ba} / \mathrm{Y}]$ values of dSph stars (e.g., Figure 14 of Tolstoy, Hill \& Tosi 2009). Such contributions seem to be missing (or muted by other contributions) in our two stars, which exhibit a subsolar ratio. This conclusion is strengthened by the upper limit on our stars' $[\mathrm{Eu} / \alpha]$ ratio $(<+0.3)$, which is lower than the super-Galactic dSph values (Figure 19 of Letarte et al. 2010) that have been ascribed to the action of AGB stars (Letarte et al. 2010).

The lower $[\mathrm{Ba} / \mathrm{Y}]$ ratio in our common proper motion pair is qualitatively consistent with the lower $r$-process component solar system $\mathrm{Ba} / \mathrm{Y}$ ratio compared to the $s$-process component as seen in Figure 11 of Burris et al. (2000), and suggests the importance of the action of the $r$-process in our two stars' chemical history. While Ba is frequently referred to as an $s$-process element, its Galactic production at $[\mathrm{Fe} / \mathrm{H}]=-1.5$ is via the $r$-process (Figure 5 of Travaglio et al. 2004a), while the lighter $n$-capture element $\mathrm{Y}$ is produced via the $r$-process and the putative light element primary process 
(LEPP; Travaglio et al. 2004a). The mean dSph ratio $[\mathrm{Ba} / \mathrm{Y}] \sim+0.60$ is in excellent agreement with the pure (i.e., no LEPP) $r$-process ratio predicted by Galactic chemical evolution models in Figure 12 of Travaglio et al. (2004a); indeed, these authors speculate that dSphs' super-Galactic pure $r$-process $[\mathrm{Ba} / \mathrm{Y}]$ ratio might be driven by a deficiency in Y caused by loss of LEPP-rich ejecta to the ISM in dSph systems. The lower $[\mathrm{Ba} / \mathrm{Y}]$ ratio in 134439/134440 might then be explained if the ISM of the parent $\mathrm{dSph}$ that birthed these 2 stars was somehow able to retain Y-rich LEPP products.

However, we discount this possibility as follows. The mean $[\mathrm{Y} / \mathrm{Fe}]$ of our two stars only differs by $\sim 0.15$ dex with respect to the mean Galactic halo field, a conclusion confirmed by additional and newer field data of Roederer et al. (2010), and lies in the midst of the scatter of dSph [Y/Fe] ratios (Venn et al. 2004). Our stars' [Ba/Fe] ratio, on the other hand, differs by 0.4-0.5 dex with respect to the Galactic halo and is at the very bottom of the scattered dSph ratios (Venn et al. 2004). It is thus $\mathrm{Ba}$, and not $\mathrm{Y}$, that is the source of our stars' unexpectedly low $[\mathrm{Ba} / \mathrm{Y}]$ value. Inasmuch as the canonical association of Eu with the $r$-process seems secure and that the limited Galactic data suggest an $r$-process origin of Ag in metal-poor stars (Crawford et al. 1998), the $n$-capture abundances consistently suggest that our stars are deficient in heavy (relative to light) $r$-process products.

Galactic halo stars evince a continuum of $r$-process abundance patterns rather than conforming to a single standard pattern (e.g., Aoki et al. 2000; Roederer et al. 2010). Indeed, observed abundance distributions have identified a significant fraction of halo stars that are deficient in heavy $r$-process elements with respect to lighter ones. Boyd et al. (2012) suggest that this "truncated r-process" may arise from nucleosynthesis via fall-back supernovae in which black hole collapse terminates the $r$-process before it runs to completion and/or prevents the dispersal of heavy nuclides into the interstellar medium. However, the progenitor mass of such supernovae are believed to be $>25 \mathrm{M}_{\odot}$ (Heger et al. 1992). This explanation for the low heavy-to-light $n$-capture ratio in HD 134439/440 is thus inconsistent with the evidence described above suggesting that these stars' nucleosynthetic history is dominated by Type II supernovae of low progenitor mass.

The schematic Galactic r-process model of Qian \& Wasserburg (2001) divides Type II SN into two classes- $\mathrm{H}$ and $\mathrm{L}$. The $\mathrm{L}$ class supernovae are less frequent events from lower mass stars that produce exclusively lighter (up to $\mathrm{Ba}$ ) $n$-capture elements; $\mathrm{H}$ events are responsible for the production of heavier $(\mathrm{A} \geq 130) n$-capture elements, but produce light $n$-capture elements as well. This phenomenological approach reproduces the Galactic $n$-capture abundance data well over the range $-3 \leq[\mathrm{Fe} / \mathrm{H}] \leq-1$ assuming no $s$-process contribution. The relative L-event yields in Figure 2 of Qian \& Wasserburg (2001) produce the Ba/Ag and Ba/Y logarithmic number abundance ratios observed in our stars to within 0.17 dex; in contrast, their Figure 2 predicts $\mathrm{Ba} / \mathrm{Y}$ ratios from $\mathrm{H}$ events some 0.80 dex larger than observed in our stars. The agreement of the light $n$-capture abundance ratios in our stars with the L-event yields is consistent with our previous conclusion that abundances in our stars are dominated by nucleosynthetic products from lower mass Type II events; the observed deficiency in some heavy $n$-capture elements in our stars is also consistent with 
this notion. Additional interpretation of the HD 134439/440 n-capture abundances in the context of the Qian \& Wasserburg (2001) L,H-event model will require measurements of other possibly highly underabundant heavy $n$-capture elements, and computational exploration of possible Levent overflow that might allow the $r$-process to reach beyond $\mathrm{A} \geq 130$ and explain the detection of any such elements.

A final possible explanation of the $n$-capture ratios in our stars that can also account for their unexpectedly high $[\mathrm{Ca}, \mathrm{Ti} / \mathrm{Fe}]$ ratios is the $\alpha$-process in a high neutron excess environment-a sce-

nario originally envisioned by Hoyle \& Fowler (1960) and first explored in detail by Woosley \& Hoffman (1992). The latter authors show that, with sufficient neutron excess, the familiar $\alpha$-rich freeze-out "merges" into what may be an (or the) $r$-process. This theoretical result is consistent with the observational work of (Roederer et al. 2010), who detect the signatures of such production in the $[\mathrm{Y} / \mathrm{Eu}]$ versus $[\mathrm{Eu} / \mathrm{Fe}]$ relation evinced by metal-poor Galactic field stars, and conclude that the r-process nucleosynthesis is "ubiquitous" in all Type II SN.

This process also naturally overproduces both $\mathrm{Ca}$ and Ti relative to other $\alpha$-elements-a signature observed in HD 134439/440 when their abundances are compared to low mass Type II progenitor yields. Moreover, the production factors calculated under two different assumptions for model zone integration in Figure 2 of Woosley \& Hoffman (1992) predict (heavy-to-light $n$-capture) $[\mathrm{Ag} / \mathrm{Y}]$ ratios that bracket the observed ratio of our stars. While nucleosynthesis in such a highentropy environment is a promising explanation for the curious $\mathrm{Ca}, \mathrm{Ti}$, and $n$-capture abundances in our stars, a rigorous examination of this hypothesis requires abundance determinations of additional $n$-capture elements in our stars and $\alpha$-process calculations extended to elements with atomic mass beyond Ag.

\section{SUMMARY}

CNO, Be, and Ag abundances are measured from high-resolution near-UV Keck/HIRES spectra for the metal-poor dwarf common proper motion pair HD 134439 and HD 134440. The CNO abundance ratios are markedly sub-solar: $[\mathrm{C} / \mathrm{Fe}]=-0.56 \pm 0.09,[\mathrm{~N} / \mathrm{Fe}]=-0.52 \pm 0.09$, and $[\mathrm{O} / \mathrm{Fe}]=-0.26 \pm 0.13$. The abundance ratios of 22 elements demonstrate a correlation with condensation temperature in our stars that is significant at the $95 \%$ confidence level. Furthermore, the differences between these abundance ratios and those characterizing the Galactic halo field at similar $[\mathrm{Fe} / \mathrm{H}]$ demonstrate correlation with $T_{\mathrm{C}}$ significant at the $\geq 99 \%$ confidence level- suggesting that the HD 134439/440 formation environment or these stars' post-formation chemical evolution differ remarkably from typical stellar denizens of the Galactic halo field.

The correlation of abundance ratios with $T_{\mathrm{C}}$ in $\mathrm{HD} 134439 / 440$ is opposite to the general trend seen in the gas-phase ISM, and instead reminiscent of a volatile-depleted refractory abundance pattern like that exhibited by primitive chondrites (e.g., Figure 1a of Yin (2005)). This pattern complements the hypothesis of Shigevama \& Tsujimoto $(2003)$, who suggest low $[\alpha / \mathrm{Fe}]$ 
halo stars originate from photospheric accretion of planetessimal material. These authors invoke stellar accretion of circumstellar Fe-rich material that would drive down $\alpha /$ Fe ratios to observed levels. While the HD 134439/440 photospheric abundance versus $T_{\mathrm{C}}$ relation is qualitatively consistent with the accretion of volatile-depleted refractory-rich material, we view this expalanation as unrealistically remarkable due to the required fine tuning of various parameters associated with the accretion process that would yield indistinguishable abundances in both components of our stellar pair.

The $U V W$ motions and low values of $[\mathrm{O}, \mathrm{Mg}, \mathrm{Si} / \mathrm{Fe}]$ for $\mathrm{HD} 134439 / 134440$ provide kinematic and chemical evidence of an extra-Galactic origin for this common proper motion pair-the low $\alpha$-element ratios being consistent in particular with an origin in a low/irregular star formation rate environment. Chen \& Zhao (2006) suggest that the abundance- $T_{\mathrm{C}}$ correlation exhibited by HD 134439/134440 can be explained by formation in an unusually dusty dSph environment. We suggest, however, that the stars' Be abundances argue against this, and that the correlation is an illusory one having a less remarkable nucleosynthetic explanation.

The majority of HD 134439/134440 abundance ratios are consistent with production from Type II SNe having low mass progenitors and/or recent chemical evolution models of dSph systems. Indeed, the $Z=0, \sim 14 \mathrm{M}_{\odot}$ Type II SNe progenitor yields of Kobayashi et al. (2006)) are able to match most of the stellar pair's observed abundance ratios; there is no evidence of Type Ia or low-to-intermediate mass star contributions to the abundance patterns. These conclusions are consistent with those reached by Shetrone et al. (2003) and Tolstoy et al. (2003) from in situ dSph abundance patterns.

The low heavy-to-light $n$-capture abundance ratio of $[\mathrm{Ba} / \mathrm{Y}] \sim-0.10$ in HD 134439/440 motivated our study of $n$-capture elements. We estimate $[\mathrm{Ag} / \mathrm{Fe}]=-0.16 \pm 0.10$ and argue that $[\mathrm{Eu} / \mathrm{Fe}]$ is significantly lower than in the similarly cool and metal-poor halo field dwarf HD 103095. Taken together, the $\mathrm{Ba}, \mathrm{Ag}$, and Eu abundances consistently suggest the heavy-to-light $n$-capture element ratio is some $\sim 0.3-0.4$ dex lower in HD 134439/134440 than in the mean Galactic halo field.

We confront the $n$-capture data with expectations from three theoretical scenarios: a) the truncated $r$-process, b) the nucleosynthetic history of HD 134439/440's precursor material being dominated by so-called L class events that produce mainly light $n$-capture elements in the phenomenological 2-component Type II SNe model of Qian \& Wasserburg (2001), and c) a significant nucleosynthetic contribution to HD 134439/134440 from material produced in an $\alpha$-rich freeze-out process in a high neutron excess environment (Woosley \& Hoffman 1992). The truncated $r$-process requires supernovae of progenitor mass higher than is compatible with the non- $n$-capture abundances in HD 134439/440. While scenarios b) and c) are consistent with the currently limited $n$-capture data, the latter is particularly attractive in that it also provides an excess production mechanism for $\mathrm{Ca}$ and $\mathrm{Ti}$ consistent with the observation that $[\mathrm{Ca}, \mathrm{Ti} / \mathrm{Fe}]>[\mathrm{O}, \mathrm{Mg}, \mathrm{Si} / \mathrm{Fe}]$ in our stars. Further constraints will require abundance determinations of additional heavy $n$-capture elements in our stars, and additional theoretical predictions concerning the production of heavy 
$n$-capture elements in these two models that can be compared to future data.

This work was supported by NSF grants AST 02-39518 and 09-08342 to J.R.K. and AST 05-05899 to A.M.B.

Facility: KECK. 


\section{REFERENCES}

Allende Prieto, C., Lambert, D. L., \& Asplund, M. 2001, ApJ, 556, L63

Anders, E., \& Grevesse, N. 1989, Geochim. Cosmochim. Acta, 53, 197

Aoki, W., Norris, J. E., Ryan, S. G., Beers, T. C. \& Ando, H. 2000, ApJ, 536, L97

Asplund, M., Grevesse, N., \& Sauval, A. J. 2005, Cosmic Abundances as Records of Stellar Evolution and Nucleosynthesis, 336, 25

Asplund, M. 2005, ARA\&A, 43, 481

Bodenheimer, P. 1966, ApJ, 144, 103

Boesgaard, A. M., King, J. R., Deliyannis, C. P., \& Vogt, S. S. 1999, AJ, 117, 492

Boesgaard, A. M., Rich, J. A., Levesque, E. M., \& Bowler, B. P. 2011, ApJ, 743, 140

Bonifacio, P., Hill, V., Molaro, P., Pasquini, L., Di Marcantonio, P., \& Santin, P. 2000, A\&A, 359, 663

Bonifacio, P., Sbordone, L., Marconi, G., Pasquini, L., \& Hill, V. 2004, A\&A, 414, 503

Boyd, R. N., Famiano, M. A., Meyer, B. S., Motizuki, Y., Kajino, T., \& Roederer, I. U. 2012, ApJ, 744, L14

Brown, J. A., Wallerstein, G., \& Zucker, D. 1997, AJ, 114, 180

Burris, D. L., Pilachowski, C. A., Armandroff, T. E., Sneden, C., Cowan, J. J., \& Roe, H. 2000, ApJ, 544, 302

Carigi, L., \& Hernandez, X. 2008, MNRAS, 390, 582

Carney, B. W., Latham, D. W., Laird, J. B., \& Aguilar, L. A. 1994, AJ, 107, 2240

Carney, B. W., Laird, J. B., Latham, D. W., \& Aguilar, L. A. 1996, 112, 668

Carney, B. W., Wright, J. S., Sneden, C., Laird, J. B., Aguilar, L. A., \& Latham, D. W. 1997, AJ, 114,363

Carretta, E., Gratton, R. G., \& Sneden, C. 2000, A\&A, 356, 238

Casagrande, L., Ramirez, I., Melendez, J., Bessell, M., \& Asplund, M. 2010, A\&A, 512, 54

Chen, Y. Q., \& Zhao, G. 2006, MNRAS, 370, 2091

Clayton, D. D. 2003, ApJ, 598, 313

Clayton, D. D. 2004, Lunar and Planetary Institute Conference Abstracts, 35, 1045 
Cohen, J. G., \& Huang, W. 2009, ApJ, 701, 1053

Crawford, J. L., Sneden, C., King, J. R., Boesgaard, A. M., \& Deliyannis, C. P. 1998, AJ, 116, 2489

De Lucia, G., Tornatore, L, Frenk, C. S., Helmi, A., Navarro, J. F., \& White, S. D. M. 2014, MNRAS, submitted (arXiv:1407.7867v1)

Demarque, P., Woo, J.-H., Kim, Y.-C., \& Yi, S. K. 2004, ApJS, 155, 667

Eggen, O. J., Lynden-Bell, D., \& Sandage, A. R. 1962, ApJ, 136, 748

Field, G.B., ApJ, 187, 453

Fitzpatrick, M. J., \& Sneden, C. 1987, BAAS, 19, 1129

Fulbright, J. P. 2002, AJ, 123, 404

Fulbright, J. P. 2000, AJ, 120, 1841

Geisler, D., Smith, V. V., Wallerstein, G., Gonzalez, G., \& Charbonnel, C. 2005, AJ, 129, 1428

Gratton, R. G., Sneden, C., Carretta, E., \& Bragaglia, A. 2000, A\&A, 354, 169

Heger, A., Fryger, C. L., Woosley, S. E., Langer, N., \& Hartmann, D. H. 1992, ApJ, 591, 288

Hopkins, P. F., Narayanan, D., \& Murray, N. 2013, MNRAS, 432, 2647

Hopkins, P. F., Keres, D., \& Murray, N. 2013, MNRAS, 432, 2639

Hoyle, F., \& Fowler, W. A. 1960, ApJ, 132, 565

Ibata, R., Lewis, G. F., Irwin, M., Totten, E., \& Quinn, T. 2001, ApJ, 551, 294

Ibata, R., Irwin, M., Lewis, G. F., \& Stolte, A. 2001, ApJ, 547, L133

Israelian, G., Ecuvillon, A., Rebolo, R., García-López, R., Bonifacio, P., \& Molaro, P. 2004, A\&A, 421,649

Ivans, I. I., Sneden, C., James, C. R., Preston, G. W., Fulbright, J. P., Höflich, P. A., Carney, B. W., \& Wheeler, J. C. 2003, ApJ, 592, 906

Johnston, K. V., Bullock, J. S., Sharma, S., Font, A., Robertson, B. E. \& Leitner, S. N. 2008, ApJ, 689,936

Jorgensen, U. G., Larsson, M., Iwamae, A., \& Yu, B. 1996, A\&A, 315, 204

King, J. R. 1997, AJ, 113, 2302

King, J. R. 1997, PASP, 109, 776 
King, J. R. 2000, AJ, 120, 1056

Kobayashi, C., Umeda, H., Nomoto, K., Tominaga, N., \& Ohkubo, T. 2006, ApJ, 653, 1145

Kraft, R. P. 1994, PASP, 106, 553

Kraft, R. P., Sneden, C., Smith, G. H., Shetrone, M. D., Langer, G. E., \& Pilachowski, C. A. 1997, AJ, 113, 279

Kraft, R. P., Sneden, C., Smith, G. H., Shetrone, M. D., \& Fulbright, J. 1998, AJ, 115, 1500

Kupka, F. G., Ryabchikova, T. A., Piskunov, N. E., Stempels, H. C., \& Weiss, W. W. 2000, Baltic Astronomy, 9, 590

Kurucz, R. L. 1995, in Astrophysical Applications of Powerful New Databases, ASPC, 78, 205

Kurucz, R. L. 2005, Memorie della Societa Astronomica Italiana Supplement, 8, 189

Lai, D. K., Johnson, J. A., Bolte, M., \& Lucatello, S. 2007, ApJ, 667, 1185

Lanfranchi, G. A., \& Matteucci, F. 2003, MNRAS, 345, 71

Lanfranchi, G. A., Matteucci, F., \& Cescutti, G. 2008, A\&A, 481, 635

Langer, G. E., \& Hoffman, R. D. 1995, PASP, 107, 177

Lejeune, T., Cuisinier, F., \& Buser, R. 1998, A\&AS, 130, 65

Letarte, B., Hill, V., Tolstoy, E., Jablonka, P., Shetrone, M., Venn, K. A., Spite, M., Irwin, M. J., Battaglia, G., Helmi, A., Primas, F., Francois, P., Kaufer, A., Szeifert, T., Arimoto, N., Sadakane, K. (2010), A\&A, 523, A17

Liu, F., Asplund, M., Ramirez, I., Yong, D., \& Melendez, J. 2014, MNRAS, 442, L51

Lodders, K. 2003, ApJ, 591, 1220

Luque, J., \& Crosley, D. R. 1999, LIFBASE: Database and spectral simulation (version 1.5), SRI International Report MP 99-009

Menci, N., Cavaliere, A., Fontana, A., Giallongo, E., \& Poli, F. 2002, ApJ, 575, 18

Monaco, L., Bellazzini, M., Bonifacio, P., Ferraro, F. R., Marconi, G., Pancino, E., Sbordone, L., \& Zaggia, S. 2005, A\&A, 441, 141

Nissen, P. E., \& Schuster, W. J. 1997, A\&A, 326, 751

Nissen, P. E., \& Schuster, W. J. 2010, A\&A, 511, L10

Penzo, C., Maccio, A. V., Casarini, L., Stinson, G. S., \& Wadsley, J. 2014, MNRAS, 442, 176 
Perryman, M. A. C., et al. 1997, A\&A, 323, L49

Pilachowski, C. A., Sneden, C., Kraft, R. P., \& Langer, G. E. 1996, AJ, 112, 545

Pillepich, A., Madau, P., \& Mayer, L. 2014, ApJ, submitted (arXiv:1407.7855v1)

Pinsonneault, M. H., Deliyannis, C. P., \& Demarque, P. 1992, ApJS, 78, 179

Pompeia, L., Hill, V., Spite, M., Cole, A., Primas, F., Romaniello, M., Pasquini, L., Cioni, M.-R., \& Smecker Hane, T. 2008, A\&A, 480, 379

Prantzos, N. 2003, Astronomical Society of the Pacific Conference Series, 304, 361

Prantzos, N. 2007, EAS Publications Series, 24, 3

Preston, G. W., \& Sneden, C. 2000, AJ, 120, 1014

Qian, Y.-Z., \& Wasserburg, G. J. 2001, ApJ, 559, 925

Ramirez, I., Asplund, M., Baumann, P., Melendez, J., \& Bensby, T. 2010, A\&A, 521, 33

Reeves, H., Fowler, W. A., \& Hoyle, F. 1970, Nature, 226, 727

Richard, O., Michaud, G. \& Richer, J. 2002, 580, 1100

Roederer, I. U., Cowan, J. J., Karakas, A. I., Kratz, K.-L., Lugaro, M., Simmerer, J., Farouqi, K., \& Sneden, C. 2010, ApJ, 724, 975

Schuler, S. C., Hatzes, A. P., King, J. R., Kürster, M., \& The, L.-S. 2006, AJ, 131, 1057

Schuler, S. C., Flateau, D., Cunha, K., King, J. R., Ghezzi, L., \& Smith, V. V. 2011, ApJ, 732, 55

Schuler, S. C., Cunha, K., Smith, V. V., Ghezzi, L., King, J. R., Deliyannis, C. P., \& Boesgaard, A. M. 2011, ApJ, 737, L32

Searle, L., \& Zinn, R. 1978, ApJ, 225, 357

Shetrone, M. D., Côté, P., \& Sargent, W. L. W. 2001, ApJ, 548, 592

Shetrone, M., Venn, K. A., Tolstoy, E., Primas, F., Hill, V., \& Kaufer, A. 2003, AJ, 125, 684

Shigeyama, T., \& Tsujimoto, T. 2003, ApJ, 598, L47

Simmerer, J., Sneden, C., Cowan, J. J., Collier, J., Woolf, V. M., \& Lawler, J. E. 2004, ApJ, 617, 1091

Smecker-Hane, T., \& Mc William, A. 1999, Spectrophotometric Dating of Stars and Galaxies, 192, 150

Smith, V. V., Cunha, K., \& Lazzaro, D. 2001, AJ, 121, 3207 
Sneden, C. 1973, ApJ, 184, 839

Spite, M., Cayrel, R., Plez, B., Hill, V., Spite, F., Depagne, E., Francois, P., Bonifacio, P. et al. 2005, A\&A, 430, 655

Stephens, A., \& Boesgaard, A. M. 2002, AJ, 123, 1647

Thielemann, F.-K., et al. 2003, Nuclear Physics A, 718, 139

Timmes, F. X., Woosley, S. E., \& Weaver, T. A. 1995, ApJS, 98, 617

Tolstoy, E., Venn, K. A., Shetrone, M., Primas, F., Hill, V., Kaufer, A., \& Szeifert, T. 2003, AJ, 125,707

Tolstoy, E., Hill, V., \& Tosi, M. 2009, ARA\&A, 47, 371

Tomkin, J., Lemke, M., Lambert, D. L., Sneden, C. 1992, AJ, 104, 1568

Travaglio, C., Gallino, R., Arnone, E., Cowan, J., Jordan, F., \& Sneden, C. 2004, ApJ, 601, 864

Travaglio, C., Hillebrandt, W., Reinecke, M., \& Thielemann, F.-K. 2004, A\&A, 425, 1029

Tsujimoto, T. 2006, A\&A, 447, 81

Venn, K. A., Irwin, M., Shetrone, M. D., Tout, C. A., Hill, V., \& Tolstoy, E. 2004, AJ, 128, 1177

Wall, J. V. \& Jenkins, C.R. 2003, Practical Statistics for Astronomers, (Cambridge, UK: Cambridge University Press), p. 64

Wheeler, J. C., Sneden, C., \& Truran, J. W., Jr. 1989, ARA\&A, 27, 279

Woosley, S. E., \& Hoffman, R. D. 1992, ApJ, 395, 202

Woosley, S. E., \& Weaver, T. A. 1995, ApJS, 101, 181

Yanny, B., Newberg, H. J., Kent, S., Laurent-Muehleisen, S. A., Pier, J. R., Richards, G. T., Stoughton, C., Anderson Jr., J. E., et al. 2000, ApJ, 540, 825

Yi, S., Demarque, P., Kim, Y.-C., Lee, Y.-W., Ree, C. H., Lejeune, T., \& Barnes, S. 2001, ApJS, 136,417

Yin, Q. 2005, ASPC, 341, 632

Zhang, L., Ishigaki, M., Aoki, W., Zhao, G., \& Chiba, M. 2009, ApJ, 706, 1095

Zinner, E. 1998, Annual Review of Earth and Planetary Sciences, 26, 147 
Table 1. Observational Data

\begin{tabular}{lcc}
\hline \hline & HD 134439 & HD 134440 \\
\hline RA $(2000)$ & $15: 10: 13.09$ & $15: 10: 12.97$ \\
Dec (2000) & $-16: 22: 45.85$ & $-16: 27: 46.52$ \\
V & 9.07 & 9.44 \\
B-V & 0.77 & 0.85 \\
Date (MJD) & 53905.314698 & 53905.325741 \\
Exposure (s) & 900 & 900 \\
$\mathrm{~S} / \mathrm{N}(\mathrm{CH})$ & 192 & 173 \\
$\mathrm{~S} / \mathrm{N}(\mathrm{NH})$ & 83 & 68 \\
$\mathrm{~S} / \mathrm{N}(\mathrm{OH})$ & 87 & 70 \\
$\pi^{\mathrm{a}}(\mathrm{mas})$ & 34.14 & 33.68 \\
$\mathrm{M}^{\mathrm{b}}\left(M_{\odot}\right)$ & 0.57 & 0.54 \\
$\mathrm{M}_{V}$ & 7.076 & 7.392 \\
$\mathrm{U}^{\mathrm{b}}(\mathrm{km} / \mathrm{s})$ & -310 & -311 \\
$\mathrm{~V}^{\mathrm{b}}(\mathrm{km} / \mathrm{s})$ & -467 & -473 \\
$\mathrm{~W}^{\mathrm{b}}(\mathrm{km} / \mathrm{s})$ & -44 & -48 \\
\hline
\end{tabular}

${ }^{a}$ From Perryman et al. (1997)

${ }^{\mathrm{b}}$ From Table 6 of Carney et al. (1994) 
Table 2. Stellar Parameters

\begin{tabular}{lcc}
\hline \hline Parameters & HD 134439 & HD 134440 \\
\hline$[\mathrm{Fe} / \mathrm{H}]^{\mathrm{a}}(\mathrm{dex})$ & $-1.47 \pm 0.07$ & $-1.53 \pm 0.09$ \\
$\mathrm{~T}_{\mathrm{eff}}{ }^{\mathrm{a}}(\mathrm{K})$ & $5000 \pm 50$ & $4785 \pm 50$ \\
$\log g(\mathrm{dex})$ & $4.69 \pm 0.10$ & $4.71 \pm 0.10$ \\
$\xi^{\mathrm{a}}(\mathrm{km} / \mathrm{s})$ & $1.5 \pm 0.3$ & $1.5 \pm 0.3$ \\
\hline
\end{tabular}

${ }^{a}$ Values from King (1997a). 
Table 3. $\quad \lambda 4324 \mathrm{CH}$ Region Linelist

\begin{tabular}{crrr}
\hline \hline $\begin{array}{c}\text { Wavelength } \\
\AA\end{array}$ & Species $^{\mathrm{a}}$ & \multicolumn{1}{c}{$\chi_{\text {low }}$} & $\log g f$ \\
$\mathrm{eV}$ & \\
\hline 4322.001 & 106.0 & 2.207 & -1.348 \\
4322.002 & 23.0 & 1.195 & -5.661 \\
4322.020 & 106.0 & 2.232 & -0.922 \\
4322.023 & 23.1 & 1.679 & -4.994 \\
4322.040 & 44.0 & 1.734 & 0.650 \\
4322.095 & 106.0 & 2.207 & -1.262 \\
4322.102 & 106.0 & 3.025 & -2.069 \\
4322.103 & 24.1 & 11.667 & -2.248 \\
4322.115 & 22.0 & 2.175 & -1.904 \\
4322.148 & 23.0 & 2.565 & -1.969 \\
4322.179 & 106.0 & 0.033 & -10.142 \\
4322.193 & 64.1 & 0.382 & -1.707 \\
4322.282 & 28.1 & 11.969 & -1.801 \\
4322.306 & 106.0 & 2.438 & -2.252 \\
4322.318 & 27.0 & 3.687 & -3.269 \\
\hline
\end{tabular}

an Tables 3-7, the species is identified by atomic number to the left of the decimal point, and ionization state (' 0 ' is neutral, ' 1 ' is singly ionized, etc) to the right. Diatomic molecules are denoted with both components' atomic numbers ('106' is $\mathrm{CH}$, '607' is $\mathrm{CN}$, etc).

Note. - A machine-readable version of the entire table is available in the online journal. A portion is shown here for guidance concerning form and content. 
Table 4. $\lambda 3330$ NH Region Linelist

\begin{tabular}{crrr}
\hline $\begin{array}{c}\text { Wavelength } \\
\AA\end{array}$ & Species & $\begin{array}{c}\chi_{\text {low }} \\
\mathrm{eV}\end{array}$ & $\log g f$ \\
\hline 3325.001 & 44.0 & 0.385 & -1.780 \\
3325.007 & 26.1 & 3.967 & -2.707 \\
3325.010 & 107.0 & 0.445 & -3.074 \\
3325.021 & 29.1 & 13.432 & -3.543 \\
3325.037 & 26.1 & 8.923 & -4.203 \\
3325.042 & 107.0 & 0.735 & -0.949 \\
3325.050 & 58.1 & 0.446 & -1.033 \\
3325.066 & 107.0 & 0.004 & -5.459 \\
3325.074 & 107.0 & 0.735 & -2.573 \\
3325.119 & 107.0 & 2.121 & -0.598 \\
3325.121 & 90.1 & 0.514 & -0.593 \\
3325.133 & 42.0 & 2.836 & -0.949 \\
3325.148 & 22.0 & 2.134 & -0.838 \\
3325.165 & 107.0 & 0.735 & -2.037 \\
3325.180 & 107.0 & 2.121 & -3.581 \\
\hline
\end{tabular}

Note. - A machine-readable version of the entire table is available in the online journal. A portion is shown here for guidance concerning form and content. 
Table 5. $\lambda 3130 \mathrm{OH}$ Region Linelist

\begin{tabular}{crrr}
\hline $\begin{array}{c}\text { Wavelength } \\
\AA\end{array}$ & Species & $\begin{array}{c}\chi_{\text {low }} \\
\mathrm{eV}\end{array}$ & $g f$ \\
\hline 3128.060 & 108.0 & 0.541 & $3.758 \mathrm{E}-03$ \\
3128.101 & 108.0 & 0.210 & $1.309 \mathrm{E}-03$ \\
3128.154 & 108.0 & 1.598 & $1.014 \mathrm{E}-04$ \\
3128.166 & 25.1 & 6.91 & $1.901 \mathrm{E}-03$ \\
3128.172 & 25.0 & 3.86 & $2.280 \mathrm{E}-04$ \\
3128.172 & 25.1 & 8.77 & $2.415 \mathrm{E}-03$ \\
3128.189 & 23.0 & 1.85 & $1.660 \mathrm{E}-06$ \\
3128.237 & 108.0 & 0.442 & $4.753 \mathrm{E}-04$ \\
3128.269 & 21.1 & 3.46 & $6.745 \mathrm{E}-01$ \\
3128.286 & 108.0 & 0.210 & $1.035 \mathrm{E}-02$ \\
3128.289 & 108.0 & 0.442 & $7.278 \mathrm{E}-04$ \\
3128.302 & 24.0 & 2.71 & $6.442 \mathrm{E}-07$ \\
3128.304 & 23.1 & 2.38 & $1.340 \mathrm{E}-01$ \\
3128.356 & 24.1 & 9.22 & $3.381 \mathrm{E}-04$ \\
3128.356 & 108.0 & 1.712 & $2.455 \mathrm{E}-03$ \\
\hline
\end{tabular}

Note. - A machine-readable version of the entire table is available in the online journal. A portion is shown here for guidance concerning form and content. 
Table 6. $\quad \lambda 3140 \mathrm{OH}$ Region Linelist

\begin{tabular}{crrr}
\hline $\begin{array}{c}\text { Wavelength } \\
\AA\end{array}$ & Species & $\begin{array}{c}\chi_{\text {low }} \\
\text { eV }\end{array}$ & $\log g f$ \\
\hline 3138.014 & 26.0 & 2.469 & -3.179 \\
3138.036 & 2.0 & 0.616 & -12.732 \\
3138.041 & 108.0 & 2.397 & -1.787 \\
3138.055 & 23.1 & 3.759 & 0.333 \\
3138.061 & 26.1 & 8.971 & 0.120 \\
3138.088 & 108.0 & 1.098 & -3.107 \\
3138.100 & 108.0 & 1.708 & -1.574 \\
3138.110 & 26.1 & 9.053 & -3.191 \\
3138.118 & 28.1 & 8.522 & -2.514 \\
3138.138 & 25.0 & 2.920 & -3.166 \\
3138.165 & 23.0 & 0.287 & -5.258 \\
3138.178 & 26.1 & 8.256 & -0.509 \\
3138.183 & 25.1 & 6.111 & -2.137 \\
3138.192 & 24.0 & 3.122 & -0.859 \\
3138.198 & 108.0 & 2.330 & -1.907 \\
\hline
\end{tabular}

Note. - A machine-readable version of the entire table is available in the online journal. A portion is shown here for guidance concerning form and content. 
Table 7. $\quad \lambda 3167$ OH Region Linelist

\begin{tabular}{crrr}
\hline $\begin{array}{c}\text { Wavelength } \\
\AA\end{array}$ & Species & $\begin{array}{c}\chi_{\text {low }} \\
\text { eV }\end{array}$ & log gf \\
\hline 3164.042 & 25.1 & 4.82 & -5.23 \\
3164.057 & 24.0 & 3.37 & -2.68 \\
3164.071 & 26.1 & 8.99 & -4.22 \\
3164.078 & 26.0 & 3.37 & -3.53 \\
3164.092 & 25.1 & 5.99 & -4.16 \\
3164.123 & 27.0 & 2.14 & -3.87 \\
3164.134 & 26.0 & 3.42 & -4.12 \\
3164.141 & 23.1 & 2.76 & -3.22 \\
3164.153 & 58.1 & 0.30 & -0.25 \\
3164.159 & 28.0 & 1.95 & -2.56 \\
3164.164 & 24.1 & 9.23 & -3.79 \\
3164.172 & 25.1 & 7.76 & -4.05 \\
3164.256 & 24.1 & 4.07 & -2.11 \\
3164.267 & 26.1 & 3.89 & -3.19 \\
3164.296 & 26.0 & 2.45 & -4.50 \\
\hline
\end{tabular}

Note. - A machine-readable version of the entire table is available in the online journal. A portion is shown here for guidance concerning form and content. 
Table 8. Carbon Abundances

\begin{tabular}{ccccc}
\hline \hline & \multicolumn{2}{c}{ HD 134439 } & \multicolumn{2}{c}{ HD 134440 } \\
$\lambda$ & $\log N(\mathrm{C})$ & {$[\mathrm{C} / \mathrm{H}]^{\mathrm{a}}$} & $\log N(\mathrm{C})$ & {$[\mathrm{C} / \mathrm{H}]^{\mathrm{a}}$} \\
$\AA$ & $\operatorname{dex}$ & $\operatorname{dex}$ & $\operatorname{dex}$ & $\operatorname{dex}$ \\
\hline 4323.02 & 6.52 & -1.87 & 6.20 & -2.19 \\
4323.22 & 6.53 & -1.86 & 6.21 & -2.18 \\
4323.50 & 6.55 & -1.84 & 6.24 & -2.15 \\
4323.85 & 6.47 & -1.92 & 6.09 & -2.30 \\
4324.12 & 6.52 & -1.87 & 6.22 & -2.17 \\
4324.40 & 6.46 & -1.93 & 6.11 & -2.28 \\
4324.81 & 6.36 & -2.03 & 6.09 & -2.30 \\
\hline
\end{tabular}

assumed solar carbon abundance of $\log N(\mathrm{C})_{\odot}=8.39 \pm 0.05 \mathrm{dex}$ Asplund et al. 2005). 
Table 9. Nitrogen Abundances

\begin{tabular}{ccccc}
\hline \hline & \multicolumn{2}{c}{ HD 134439 } & \multicolumn{2}{c}{ HD 134440 } \\
$\lambda$ & $\log N(\mathrm{~N})$ & {$[\mathrm{N} / \mathrm{H}]^{\mathrm{a}}$} & $\log N(\mathrm{~N})$ & {$[\mathrm{N} / \mathrm{H}]^{\mathrm{a}}$} \\
$\AA$ & $\operatorname{dex}$ & $\operatorname{dex}$ & $\operatorname{dex}$ & $\operatorname{dex}$ \\
\hline 3326.41 & 5.84 & -1.94 & 5.76 & -2.02 \\
3328.20 & 5.84 & -1.94 & 5.65 & -2.13 \\
3330.27 & 5.79 & -1.99 & 5.61 & -2.17 \\
3330.92 & 5.83 & -1.95 & 5.70 & -2.08 \\
\hline
\end{tabular}

a Assumed solar nitrogen abundance of $\log N(\mathrm{~N})_{\odot}=7.78 \pm 0.06$ dex Asplund et al. 2005). 
Table 10. Oxygen Abundances

\begin{tabular}{ccccc}
\hline \hline & \multicolumn{2}{c}{ HD 134439 } & \multicolumn{2}{c}{ HD 134440 } \\
$\lambda$ & $\log N(\mathrm{O})$ & {$[\mathrm{O} / \mathrm{H}]^{\mathrm{a}}$} & $\log N(\mathrm{O})$ & {$[\mathrm{O} / \mathrm{H}]^{\mathrm{a}}$} \\
$\AA$ & $\operatorname{dex}$ & $\operatorname{dex}$ & $\operatorname{dex}$ & $\operatorname{dex}$ \\
\hline 3129.94 & 7.11 & -1.58 & 6.98 & -1.71 \\
3138.78 & 6.99 & -1.70 & 6.76 & -1.93 \\
3138.92 & 6.93 & -1.76 & 6.64 & -2.05 \\
3139.30 & 7.07 & -1.62 & 6.73 & -1.96 \\
3140.51 & 7.06 & -1.63 & 6.95 & -1.74 \\
3141.66 & 6.96 & -1.73 & 6.93 & -1.76 \\
3167.17 & 7.03 & -1.66 & 6.79 & -1.90 \\
\hline
\end{tabular}

${ }^{a}$ Assumed solar oxygen abundance of $\log N(\mathrm{O})_{\odot}=8.69 \pm 0.05 \operatorname{dex}$ (Allende Prieto et al. 2001). 
Table 11. Abundance Sensitivities and Uncertainties

\begin{tabular}{ccccccc}
\hline \hline & $\Delta \mathrm{T}_{\text {eff }}$ & $\Delta \log g$ & $\Delta \xi$ & $\Delta$ cont $^{\mathrm{a}}$ & $\sigma^{\mathrm{b}}$ & $\sigma_{\text {avg }}{ }^{\mathrm{c}}$ \\
& $\pm 150 \mathrm{~K}$ & $\pm 0.20 \mathrm{dex}$ & $\pm 0.5 \mathrm{~km} / \mathrm{s}$ & $\operatorname{dex}$ & $\operatorname{dex}$ & $\operatorname{dex}$ \\
\hline$\Delta[\mathrm{C} / \mathrm{Fe}]$ & \pm 0.09 & $\mp 0.04$ & \pm 0.03 & 0.04 & 0.07 & 0.09 \\
$\Delta[\mathrm{N} / \mathrm{Fe}]$ & \pm 0.12 & $\mp 0.05$ & \pm 0.02 & 0.06 & 0.05 & 0.09 \\
$\Delta[\mathrm{O} / \mathrm{Fe}]$ & \pm 0.15 & $\mp 0.06$ & \pm 0.00 & 0.06 & 0.10 & 0.13 \\
\hline
\end{tabular}

${ }^{a}$ This is the abundance uncertainty arising from the uncertainty in setting the pseudo-continuum of the spectra.

${ }^{\mathrm{b}} \sigma$ is the statistical uncertainty in the mean abundance from the molecular lines measured.

${ }^{\mathrm{c}}$ This is the final total uncertainty in the $[\mathrm{X} / \mathrm{Fe}]$ ratios for $\mathrm{HD}$ 134439 and HD 134440.

Note. - We have adopted the sensitivities of $[\mathrm{Fe} / \mathrm{H}]$ due to $\mathrm{T}_{\text {eff }}$, $\log g, \xi$, and statistical uncertainty from Table 3 of King (1997a) for the calculations in this table. 
Table 12. Elemental Abundances

\begin{tabular}{|c|c|c|c|c|c|c|}
\hline & $\mathrm{X}$ & $\begin{array}{c}\mathrm{X} / \mathrm{Fe}] \\
\text { HD } 134439\end{array}$ & $\begin{array}{c}\mathrm{X} / \mathrm{Fe}] \\
\text { HD } 134440\end{array}$ & {$[\mathrm{X} / \mathrm{Fe}]_{\text {avg }}{ }^{\mathrm{a}}$} & $\begin{array}{c}{[\mathrm{X} / \mathrm{Fe}]_{\text {Halo }}} \\
{[\mathrm{Fe} / \mathrm{H}]=-1.5}\end{array}$ & 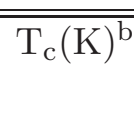 \\
\hline & $\mathrm{C}$ & -0.43 & -0.70 & $-0.56 \pm 0.09$ & -0.12 & 40 \\
\hline & $\mathrm{N}$ & -0.48 & -0.57 & $-0.52 \pm 0.09$ & +0.00 & 123 \\
\hline \multirow{5}{*}{$\begin{array}{c}\alpha \\
\text { elements }\end{array}$} & $\mathrm{O}$ & -0.20 & -0.33 & $-0.26 \pm 0.13$ & +0.54 & 180 \\
\hline & $\mathrm{Mg}$ & -0.09 & -0.10 & $-0.10 \pm 0.05$ & +0.39 & 1336 \\
\hline & $\mathrm{Si}$ & +0.04 & +0.04 & $+0.04 \pm 0.10$ & +0.30 & 1310 \\
\hline & $\mathrm{Ca}$ & +0.09 & +0.08 & $+0.08 \pm 0.04$ & +0.31 & 1517 \\
\hline & $\mathrm{Ti}$ & +0.18 & +0.20 & $+0.19 \pm 0.09$ & +0.24 & 1582 \\
\hline \multirow{8}{*}{$\begin{array}{c}\text { odd Z } \\
\text { elements }\end{array}$} & $\mathrm{Na}$ & -0.48 & -0.49 & $-0.48 \pm 0.06$ & -0.16 & 958 \\
\hline & $\mathrm{Al}$ & -0.22 & -0.19 & $-0.20 \pm 0.04$ & -0.31 & 1653 \\
\hline & $\mathrm{K}$ & +0.10 & +0.08 & $+0.09 \pm 0.08$ & +0.50 & 1006 \\
\hline & $\mathrm{Sc}$ & -0.03 & -0.04 & $-0.04 \pm 0.07$ & -0.09 & 1659 \\
\hline & $\mathrm{V}$ & +0.01 & +0.05 & $+0.03 \pm 0.08$ & +0.08 & 1429 \\
\hline & Mn & -0.41 & -0.37 & $-0.39 \pm 0.05$ & -0.45 & 1158 \\
\hline & $\mathrm{Co}$ & -0.03 & +0.05 & $+0.01 \pm 0.05$ & -0.11 & 1352 \\
\hline & $\mathrm{Cu}$ & -0.69 & $\ldots$ & $\ldots$ & -0.57 & 1037 \\
\hline \multirow{4}{*}{$\begin{array}{c}\text { even Z } \\
\text { elements }\end{array}$} & $\mathrm{Fe}^{\mathrm{c}}$ & $(-1.47)$ & $(-1.53)$ & $(-1.50 \pm 0.09)$ & +0 & 1334 \\
\hline & $\mathrm{Cr}$ & -0.01 & +0.06 & $+0.02 \pm 0.06$ & -0.04 & 1296 \\
\hline & $\mathrm{Ni}$ & -0.12 & -0.13 & $-0.12 \pm 0.05$ & -0.06 & 1353 \\
\hline & $\mathrm{Zn}$ & -0.04 & -0.06 & $-0.05 \pm 0.10$ & +0.06 & 726 \\
\hline \multirow{3}{*}{$\begin{array}{l}\text { n-capture } \\
\text { elements }\end{array}$} & $\mathrm{Y}$ & -0.22 & -0.28 & $-0.25 \pm 0.08$ & -0.07 & 1659 \\
\hline & $\mathrm{Ag}$ & -0.19 & -0.13 & $-0.16 \pm 0.10$ & +0.28 & 996 \\
\hline & $\mathrm{Ba}$ & -0.35 & -0.36 & $-0.36 \pm 0.07$ & +0.05 & 1455 \\
\hline
\end{tabular}

$\mathrm{a}[\mathrm{X} / \mathrm{Fe}]_{\text {avg }}$ is the average $[\mathrm{X} / \mathrm{Fe}]$ of HD 134439 and HD 134440.

${ }^{\mathrm{b}}$ Condensation temperatures are taken from Table 8 of Lodders (2003).

${ }^{c}$ These are $[\mathrm{Fe} / \mathrm{H}]$ values from King (1997a).

Note. - Elemental abundances and uncertainties, with the exception of C, N, O, Fe, 
and Ag are taken from Chen \& Zhao (2006). 


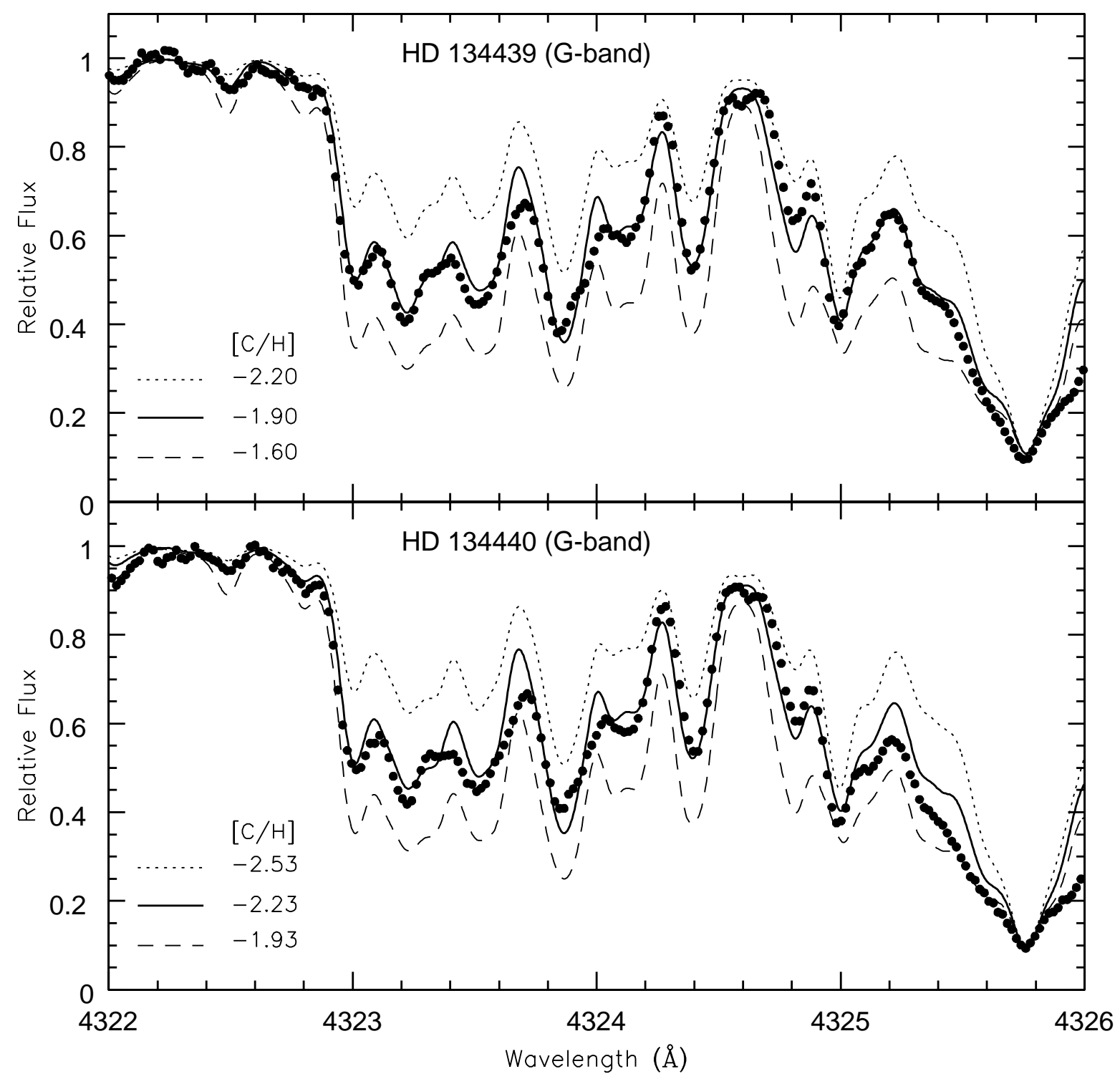

Fig. 1.- G-band synthesis for HD 134439 (upper panel) and HD 134440 (lower panel). Filled circles indicate observed spectra. The solid line is the average best-fit $[\mathrm{C} / \mathrm{H}]$ abundance determined by $\chi^{2}$-tests on individual lines. Dotted and dashed lines are \pm 0.30 dex deviations from the best-fit abundance. 


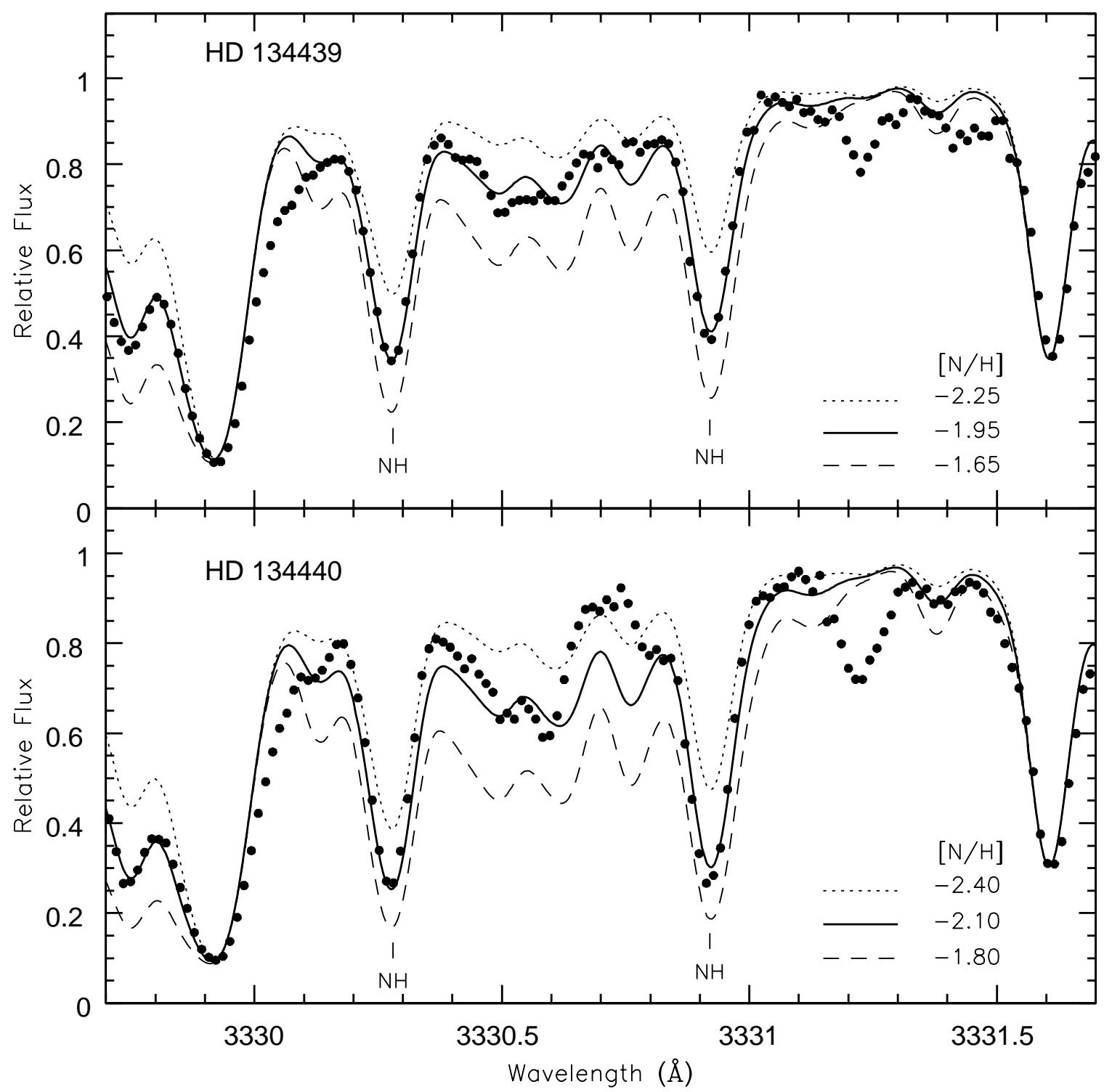

Fig. 2.- Synthesis of NH molecular lines for HD 134439 (upper panel) and HD 134440 (lower panel). Filled circles indicate observed spectra. The solid line is the average best-fit $[\mathrm{N} / \mathrm{H}]$ abundance determined by $\chi^{2}$-test on the individual $\mathrm{NH}$ lines indicated. Dotted and dashed lines are \pm 0.30 dex deviations from the best-fit abundance. 


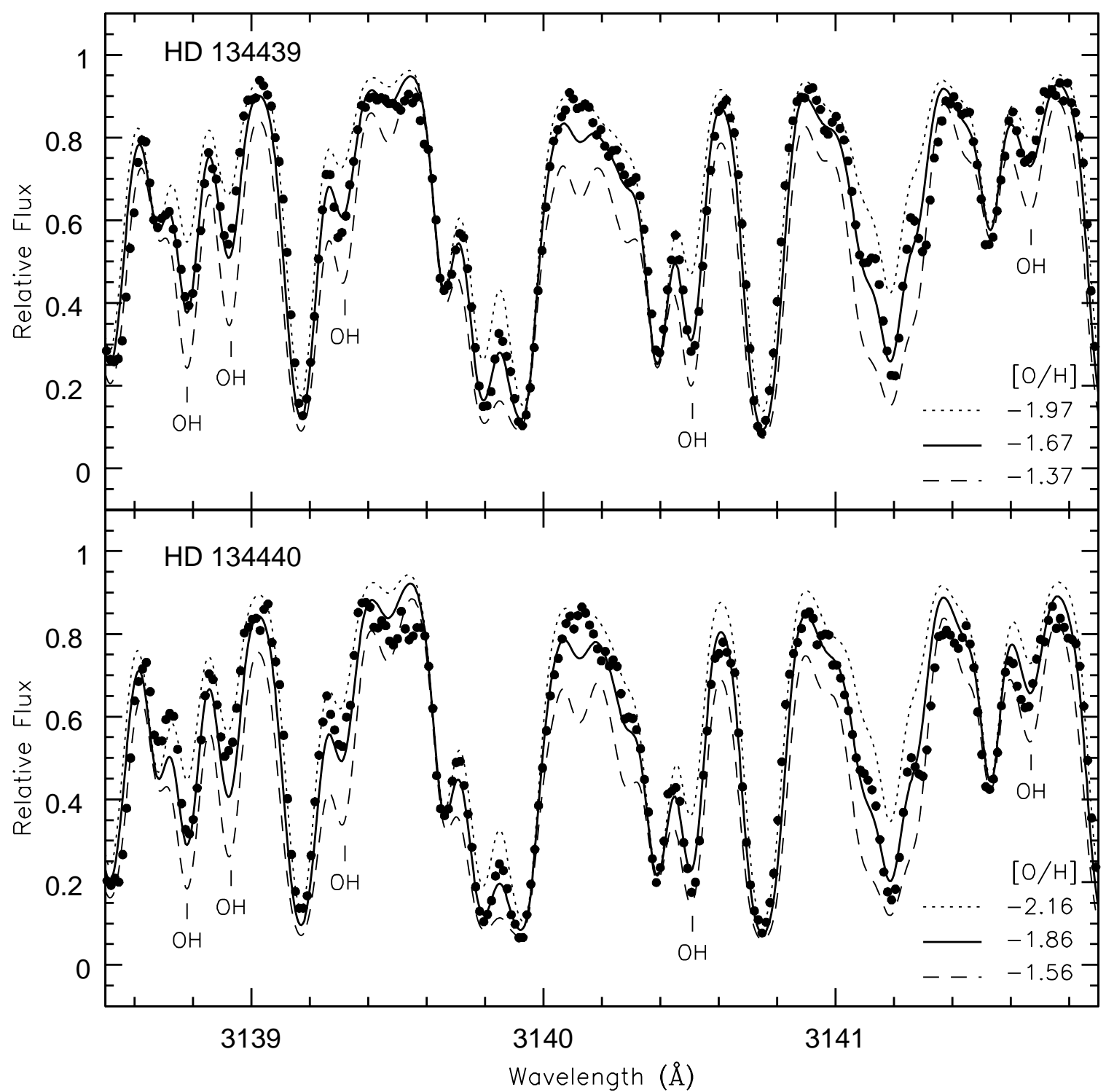

Fig. 3.- Synthesis of OH molecular lines for HD 134439 (upper panel) and HD 134440 (lower panel). Filled circles indicate observed spectra. The solid line is the average best-fit $[\mathrm{O} / \mathrm{H}]$ abundance determined by $\chi^{2}$-test on all the $\mathrm{OH}$ lines considered. Dotted and dashed lines are \pm 0.30 dex deviations from the best-fit abundance. 


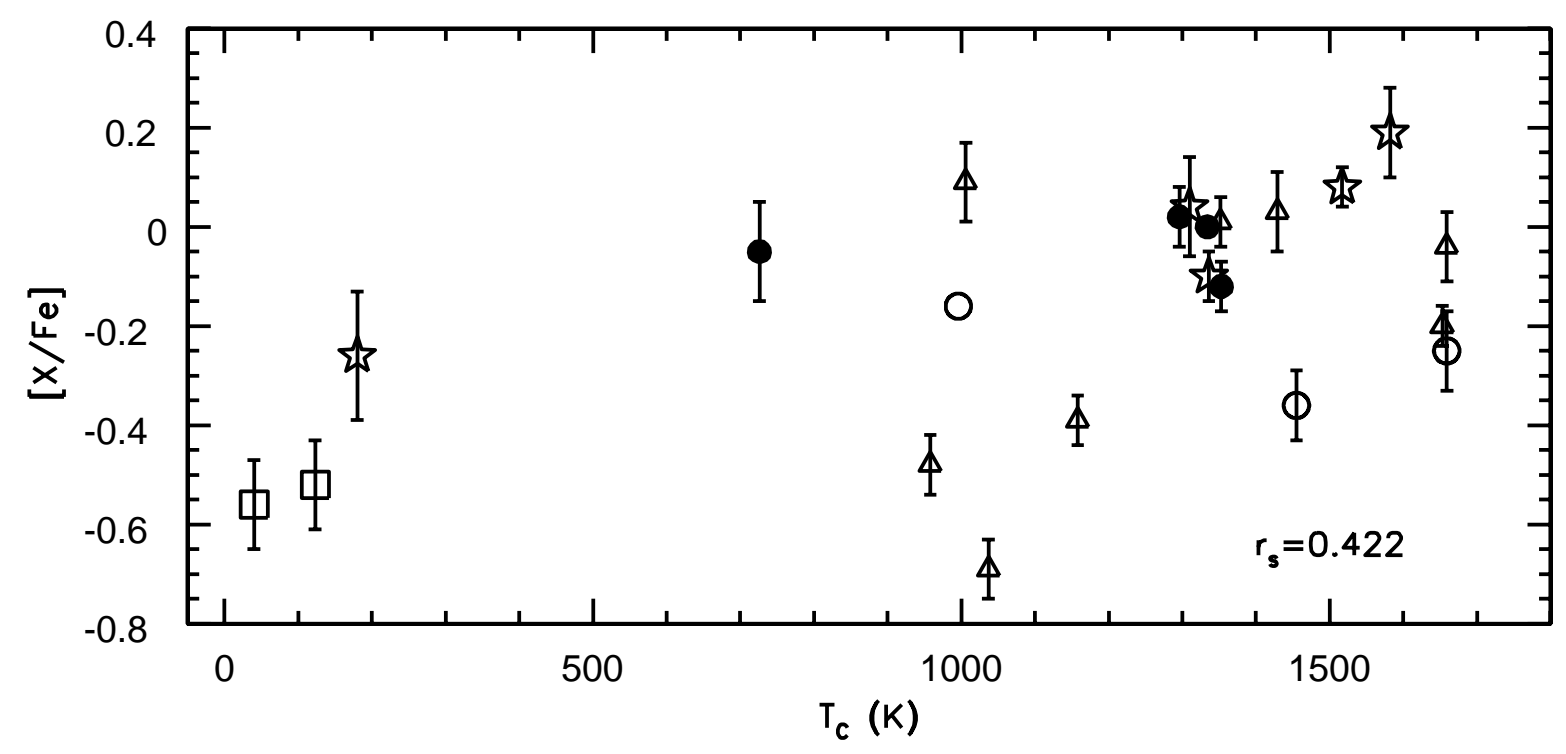

Fig. 4.- Mean [X/Fe] of HD 134439 and HD 134440 for the 22 elements in Table 12 versus condensation temperature $T_{\mathrm{C}}$. The open star symbols indicate $\alpha$ elements, triangles designate odd Fe group elements, filled circles are for even iron group elements, open circles are for $n$-capture elements, and open squares are $\mathrm{C}$ and $\mathrm{N}$. One $\sigma$ error bars for the mean abundance ratios are shown. The Spearman rank correlation coefficient for the data is 0.422 . 


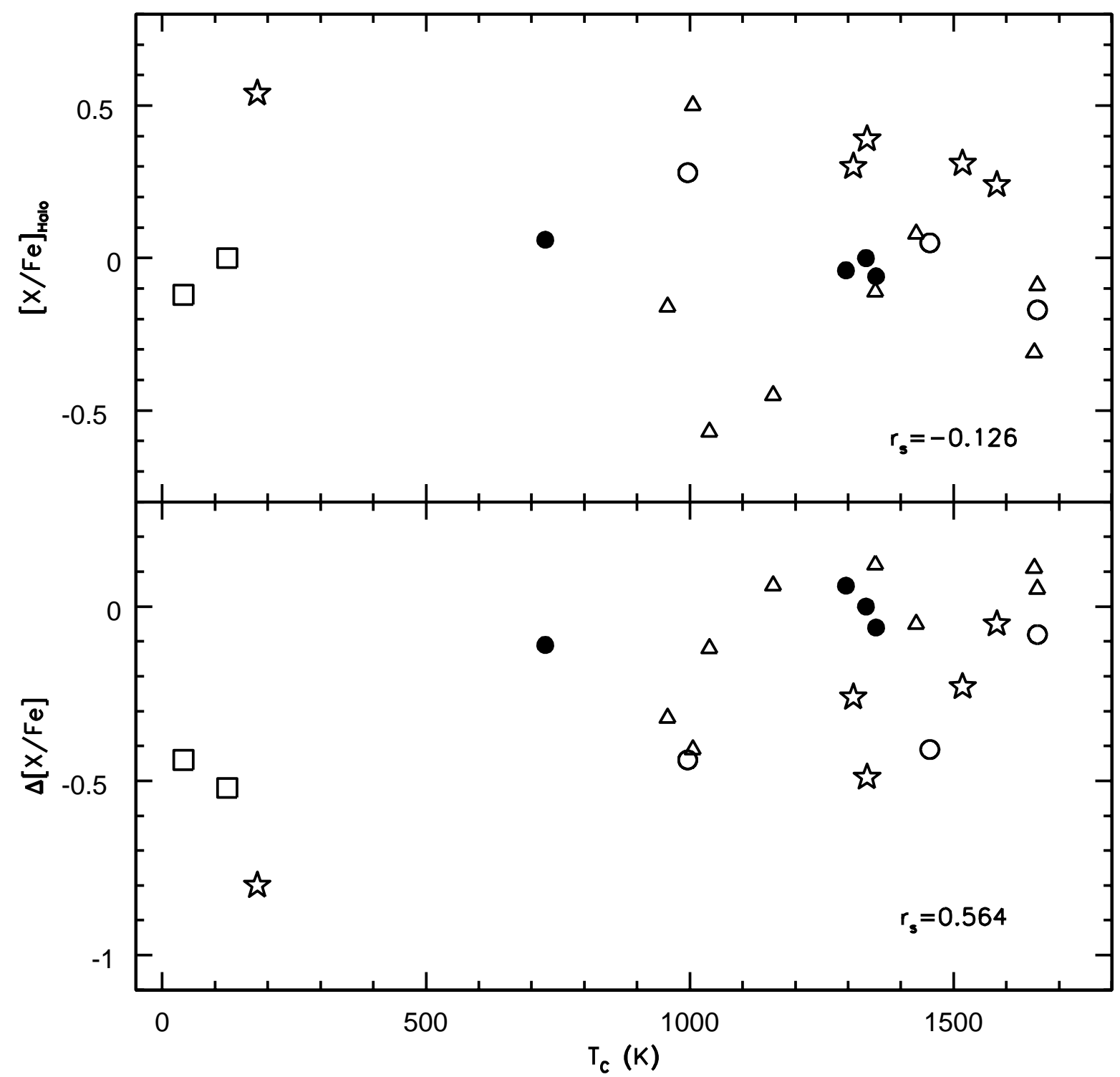

Fig. 5.- Mean $[\mathrm{X} / \mathrm{Fe}]$ for Galactic halo field stars at $[\mathrm{Fe} / \mathrm{H}] \sim-1.50$ (see text) are plotted against $T_{\mathrm{C}}$ in the upper panel. The lower panel shows the differences between mean galactic halo $[\mathrm{X} / \mathrm{Fe}]$ and values of HD 134439 and 134440 (in the sense our stars minus halo stars). The symbols are as in Figure 4 


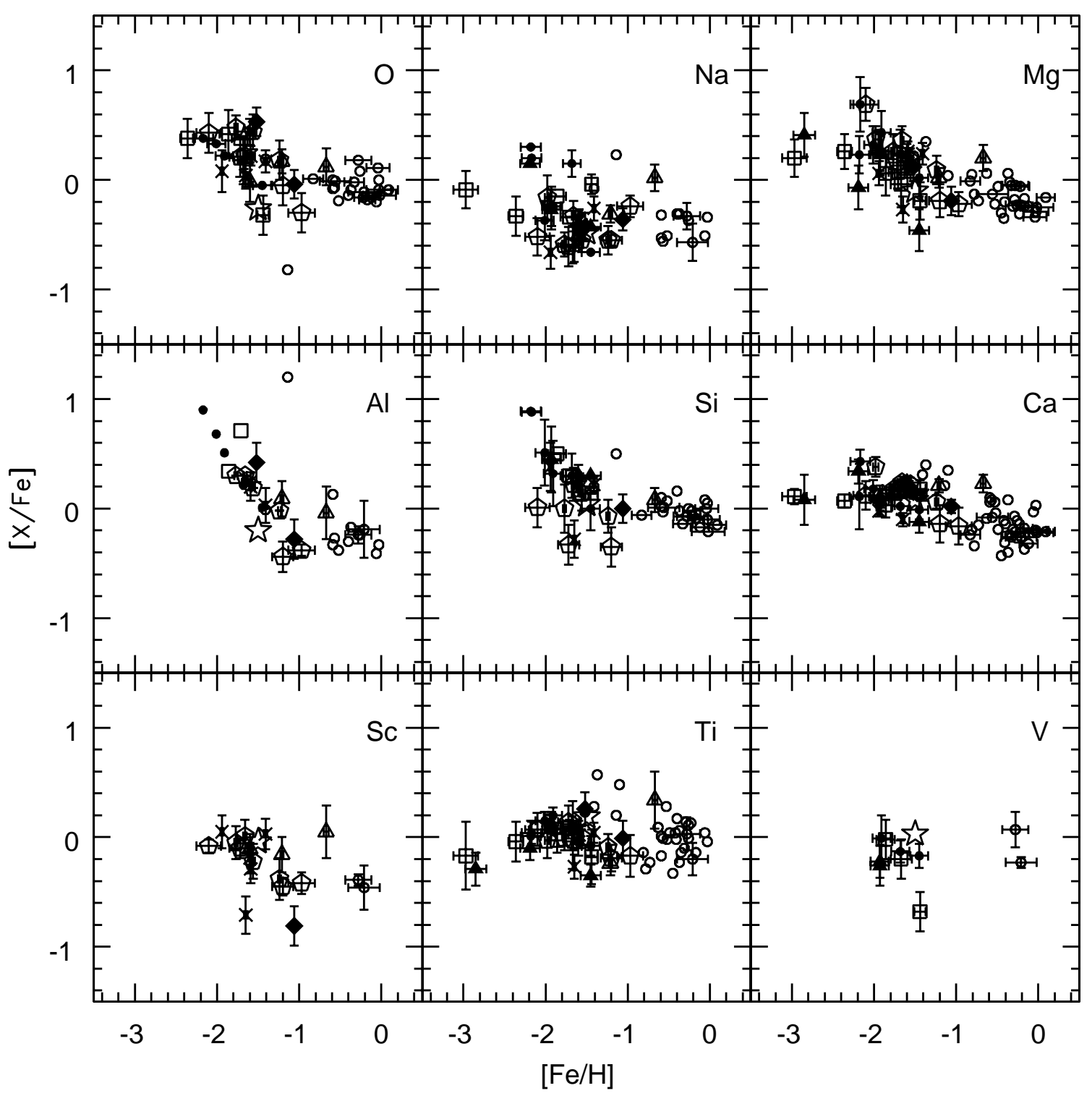

Fig. 6. - $[\mathrm{X} / \mathrm{Fe}]$ as a function $[\mathrm{Fe} / \mathrm{H}]$ for stars in $\mathrm{dSph}$ galaxies from various studies (see text). The dSph galaxies are shown in different symbols: open circles for Sgr, open pentagons for Scl, open squares for Dra, open triangles for For, filled circles for UMi, filled triangles for Sex, filled diamonds for Leo, and crosses for Car. The open star symbol indicates the mean [X/Fe] for HD 134439 and HD 134440. 


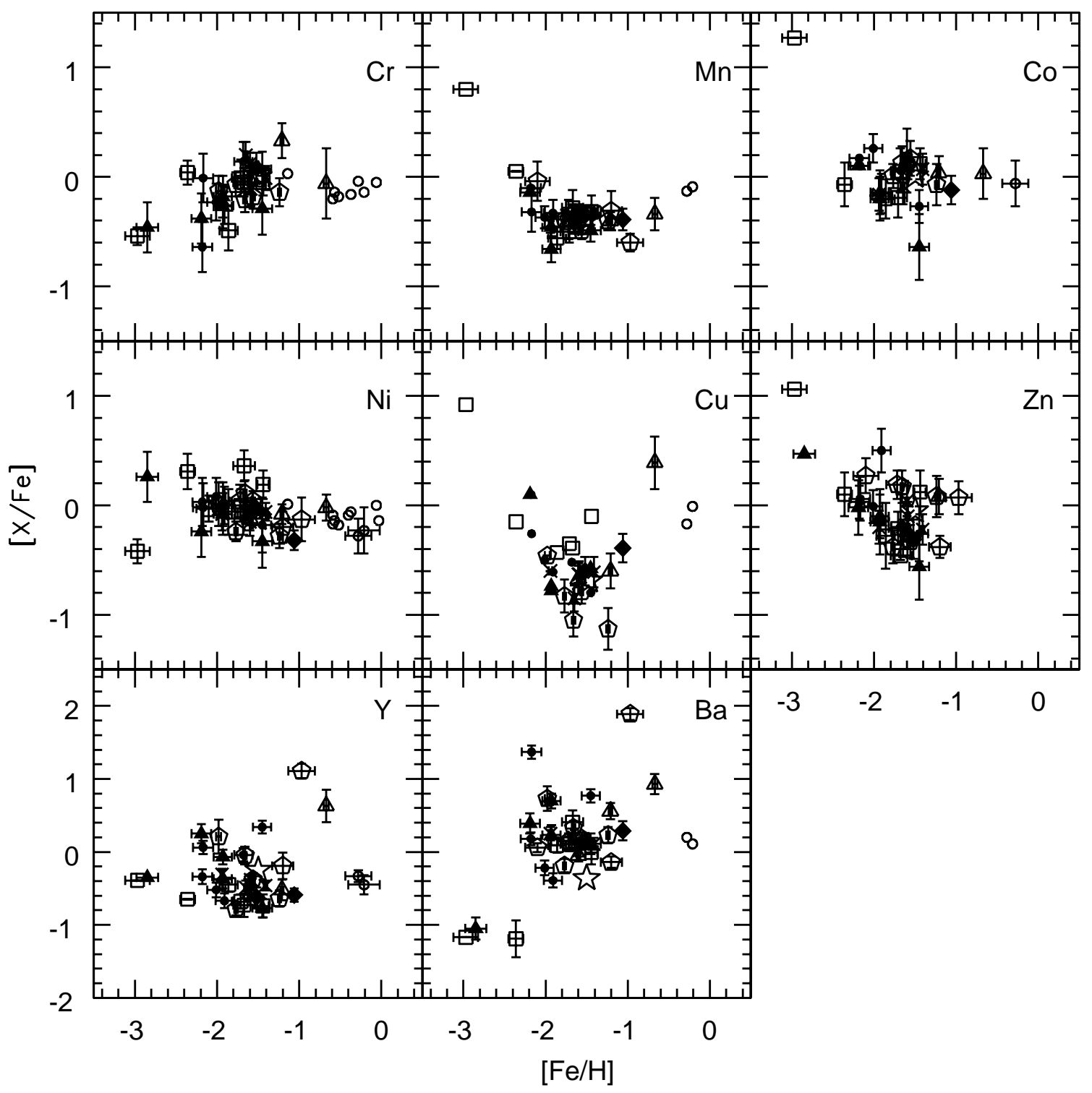

Fig. 7.- Same as Figure 6 for additional element ratios. 


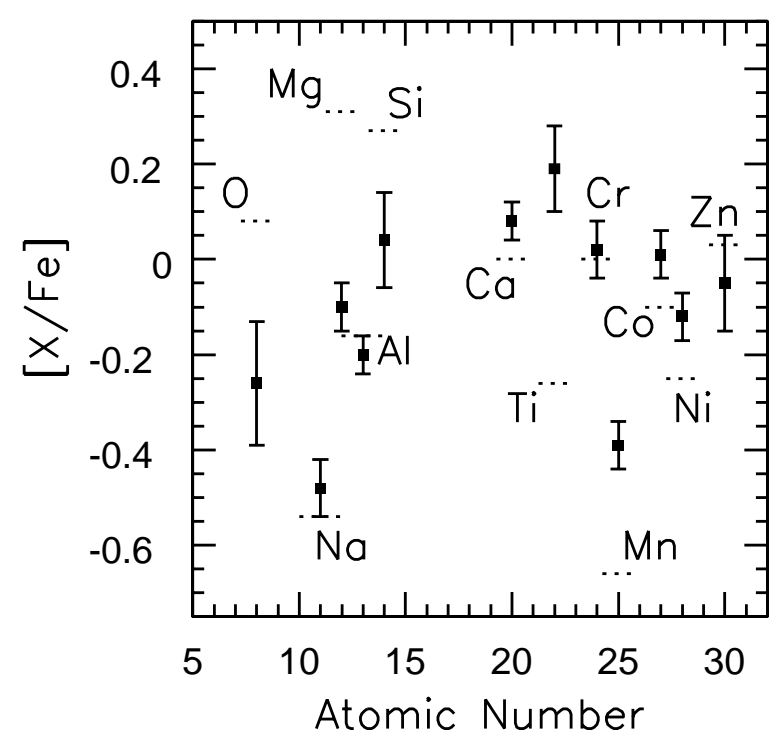

Fig. 8.- Observed mean $[\mathrm{X} / \mathrm{Fe}]$ ratios for HD 134439/134440 (data points) compared to the abundance ratios from the $Z=0, M=14 \mathrm{M}_{\odot}$ progenitor models of Kobayashi et al. (2006; horizontal dashed lines. 

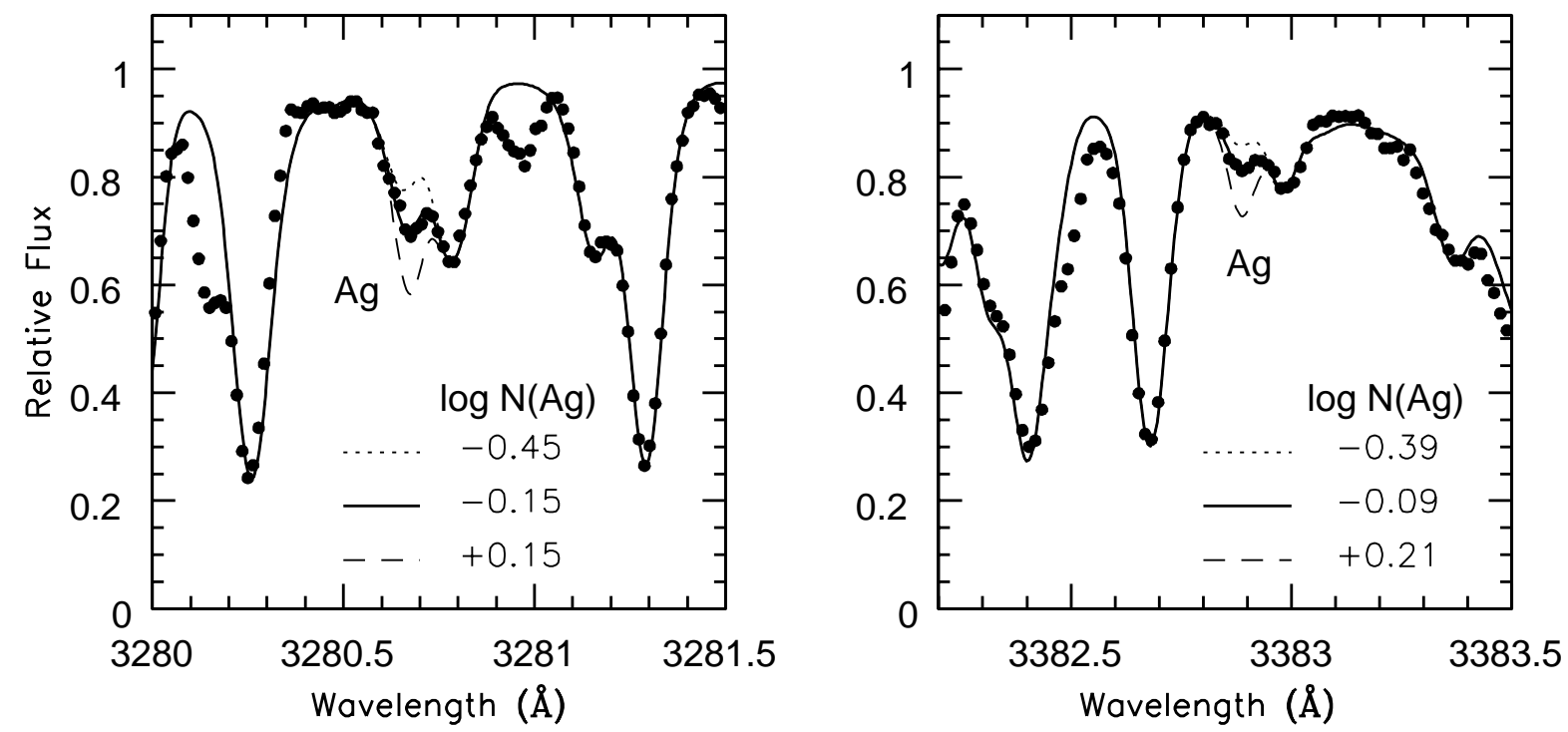

Fig. 9. - Syntheses of Ag I features at $3280 \AA$ (left panel) and $3382 \AA$ (right panel) for HD 134439. Filled circles indicate observed spectra. The solid line is the best fit Ag abundance; dotted and dashed lines are \pm 0.30 dex deviations from the best fit abundance. 

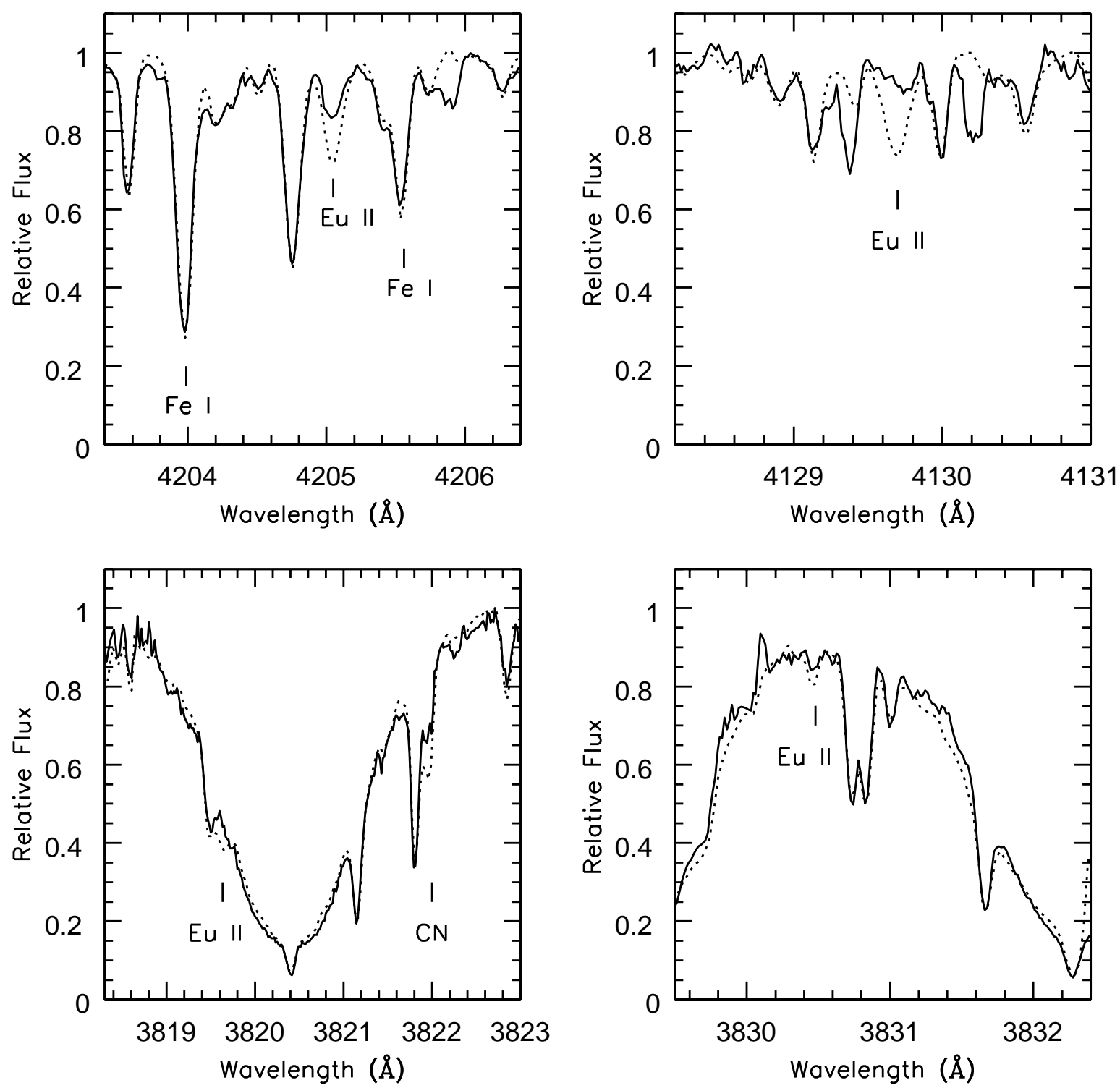

Fig. 10. - A comparison of 4 different Eu II features in consistently normalized observed spectra of HD 134439 (solid line) and HD 103095 (dotted line). The Eu lines in HD 103095 are consistently stronger relative to neighboring metal lines compared to HD 134439. The feature near $4130.2 \AA$ is believed to be an artifact in the HD 134439 spectrum. The feature near 4129.4A, however, appears real; Yb II lines are in present in the VALD list at this wavelength region, but we do not observe what should be stronger known Yb II features at other wavelengths. 